\title{
Mrk 609: resolving the circumnuclear structure with near-infrared integral field spectroscopy ${ }^{\star}$
}

\author{
J. Zuther ${ }^{1}$, C. Iserlohe ${ }^{1}$, J.-U. Pott ${ }^{1,2}$, T. Bertram ${ }^{1}$, S. Fischer ${ }^{1}$, W. Voges ${ }^{3}$, G. Hasinger ${ }^{3}$, and A. Eckart ${ }^{1}$ \\ 1 I. Physikalisches Institut, Universität zu Köln, Zülpicher Str. 77, 50937 Köln, Germany \\ e-mail: zuther@ph1.uni-koeln.de \\ 2 European Southern Observatory (ESO), Garching bei München, Germany \\ 3 Max-Planck Institut für extraterrestrische Physik, Garching bei München, Germany
}

Received 25 April 2006 / Accepted 21 February 2006

\begin{abstract}
Aims. We present first results of near-infrared (NIR) $J$ and $H+K$ ESO-SINFONI integral field spectroscopy of the composite starburst/Seyfert 1.8 galaxy Mrk 609. The data were taken during the science verification period of SINFONI. We aim to investigate the morphology and excitation conditions within the central $2 \mathrm{kpc}$. Additional Nobeyama $45 \mathrm{~m} \mathrm{CO}(1-0)$ data are presented, which we used to estimate the molecular gas mass. The source was selected from a sample of SDSS/ROSAT-based, X-ray bright AGN with redshifts of $0.03<z<1$ that are suitable for adaptive optics observations. This sample allows for a detailed study of the NIR properties of the nuclear and host environments with high spectral and spatial resolution.

Methods. Integral field spectroscopy with SINFONI delivers simultaneous spatial and spectral coverage of the circumnuclear environment. The NIR light is influenced less by dust extinction than by optical light and is sensitive to mass- dominating stellar populations. Furthermore, several NIR emission lines allow us to distinguish between Seyfert and starburst activities.

Results. Our NIR data reveal a complex emission-line morphology that is possibly associated with a nuclear bar seen in the reconstructed continuum images. The detections of [Si VI] and a broad Pa $\alpha$ component are clear indicators of the presence of an accreting super-massive black hole at the center of Mrk 609. In agreement with previous observations, we find that the circumnuclear emission is not significantly extincted. Analysis of the high angular-resolution, molecular hydrogen emission and [Fe II] emission reveals the LINER character of the nucleus. The large $\mathrm{H}_{2}$ gas mass deduced from the $\mathrm{CO}(1-0)$ observation provides the fuel needed to feed the starburst and Seyfert activity in Mrk 609.

Conclusions. High angular resolution imaging spectroscopy provides an ideal tool for resolving the nuclear and starburst contributions in active galaxies. We show that Mrk 609 exhibits LINER features that appear to be hidden in visible/NIR spectra with larger apertures.
\end{abstract}

Key words. galaxies: Seyfert - galaxies: starburst - galaxies: fundamental parameters - infrared: galaxies galaxies: individual: Mrk 609

\section{Introduction}

Stellar kinematical studies of nearby galaxies give grounds speculating that every galaxy harbors a super-massive black hole (SMBH) in its center (e.g. Kormendy \& Richstone 1995; Gebhardt et al. 2000; Tremaine et al. 2002). Numerous studies of active galactic nuclei (AGN) and quiescent galaxies have furthermore revealed a correlation between the SMBHs and the bulges hosting them (e.g. Magorrian et al. 1998; Page et al. 2001; Tremaine et al. 2002). These correlations indicate the existence of feedback mechanisms that regulate the coeval growth of SMBHs and their associated bulges.

In this paper we present results of an NIR study of the composite galaxy Mrk 609 (Fig. 1 and Zuther et al. 2006) with the new adaptive-optics (AO) assisted integral field spectrometer (IFS) SINFONI at the ESO VLT. For the first time, AO-assisted imaging spectroscopy on 8-10 m class telescopes allows for the simultaneous study of the morphology, chemical composition, and kinematics of the circumnuclear regions of AGN at an unprecedented spatial resolution.

* Based on ESO-VLT SINFONI science verification 60.A-9041(A) and Nobeyama Radio Observatory $45 \mathrm{~m}$ telescope observations.

\subsection{Fueling of nuclear activity}

The presence of the Seyfert phenomenon is supposed to originate in the accretion of matter onto an SMBH in the centers of the galaxies. The fuel necessary for driving nuclear activity, which is composed of nuclear starbursts and Seyfert-like activity, has to be transported from galactic scales $(\sim 10 \mathrm{kpc})$ down to nuclear scales of $\sim 10 \mathrm{pc}$. We are still far from understanding the detailed processes that lead to the dissipation of angular momentum needed for the gas and stars to fall towards the nuclear region. However, considerable theoretical, as well as observational, effort has been made toward understanding these processes (e.g. Shlosman et al. 1990; Combes 1996; Knapen 2005). External and internal triggers of the fueling process can be distinguished.

External triggers are related to the environment of galaxies and gravitational interaction. Non-axisymmetries, which can lead to loss of angular momentum, can result from galaxy interactions. For ULIRGs, which show the most extreme case of infrared nuclear activity, there is intriguing evidence of a connection between galaxy interaction and nuclear activity (cf. review by Knapen 2005, and references therein). Nevertheless, statistical studies show a significant fraction of nuclear active galaxies 
that are apparently free of any external trigger. Among the internal triggers, for example, Shlosman et al. (1989) proposed a two-step process that is able to sweep the interstellar medium (ISM) via a stellar bar from large scales into a disk of several hundred pc in radius. In the second step, further instabilities (barwithin-bar) drive the material close to the nucleus until viscous processes take over the angular momentum transport.

While extensive star formation in quiescent galaxies appears to be related to large-scale bars (Knapen 2005, and references therein), the observational evidence of bar-related nuclear activity is not as clear for Seyfert galaxies. Laine et al. (2002) find a slight but significant increase in the galactic-bar fraction of active galaxies when compared to in-active galaxies. This does not appear to be the case for nuclear bars. On the other hand, there are a considerable number of AGN that show no signs of any bar or inactive galaxies that do possess bars. The overall lack of observational evidence of direct causal relationships between the presence of morphological asymmetries and the starburst/Seyfert activity might result from disregarding the correct spatial or time scales (Knapen 2005). To some extent the non-axisymmetries could occur on spatial scales that are currently not resolvable, or could be masked by dust or star formation (Laine et al. 2002). Considering lifetimes, the typical age for nearby spiral galaxies is $10^{10} \mathrm{yr}$. Only $\sim 10 \%$ of nearby spirals display Seyfert activity (e.g. Ho et al. 1997). Sanders et al. (1988) propose an evolutionary scenario in which the Seyfert phenomenon is a transient phase. If all galaxies undergo this phase, the small fraction of active galaxies translates into an AGN lifetime of about $10^{9}$ yr. But the non-stellar activity can also occur episodically on much shorter time scales (Martini 2004). The typical bar lifetime is $10^{9} \mathrm{yr}$ (Crenshaw et al. 2003), and the bar can trigger significant star formation within $10^{8}$ yr (Hunt \& Malkan 1999). Active circumnuclear star formation might, therefore, be related to the presence of a bar. But what happens with the bar when the Seyfert activity, under the assumption of bar-induced nuclear fueling, is episodic with a lifetime of about $10^{4} \mathrm{yr}$ ? Further work has clearly to be done, primarily aimed at the kinematics and dynamics of the very central region of active and inactive galaxies.

The recent finding of a stellar surface mass density criterion for the separation of bulge-dominated and disk-dominated galaxies by Kauffmann et al. (2003) can shed some light on the problem. The authors find that bulges dominate in stellar masses $\gtrsim 3 \times 10^{10} M_{\odot}$ corresponding to a mean stellar surface density of $\mu_{\star} \gtrsim 3 \times 10^{8} M_{\odot} \mathrm{kpc}^{-2}$. Heckman et al. (2004) find that this stellar surface density is also the critical surface density for the onset of significant Seyfert activity. These results can be compared with the critical stellar surface density necessary for the onset of bar instability (Wyse 2004). It turns out that this surface density $\left(\mu>1.7 \times 10^{8} M_{\odot} \mathrm{kpc}^{-2}\right)$ is very similar to the one found by Heckman et al. (2004) for nuclear activity. One expects that the stellar surface density increases once a bar is formed, since the subsequent gas infall towards the central regions will be accompanied by star formation. This is seen in both observations and in simulations and, thus, can explain the factor of about 2 below the observed stellar surface density for bulge dominance.

\subsection{Starburst/Seyfert composite galaxies}

A subgroup of AGN, the starburst/Seyfert composite galaxies (Moran et al. 1996), appears to be best suited to studying the starburst-AGN connection, since the AGN and starburst components present themselves at the same level of activity (Panessa et al. 2005). These objects can be characterized by optical spectra that are dominated by starburst features, while the Xray luminosity and its variability are typical of Seyfert galaxies. The former property is based on the emission-line diagnostic diagrams by Veilleux \& Osterbrock (1987). Close inspection of the optical spectra often reveals some weak Seyfert-like features, e.g. [O III] being significantly broader than all other narrow lines or a weak broad $\mathrm{H} \alpha$ component. There is a resemblance to narrow-line X-ray galaxies (NLXG, e.g. Boyle et al. 1995), which also show spectra of a composite nature. Their soft X-ray spectra are hard, i.e. flat (Almaini et al. 1996), but it is still not clear how this strong and hard X-ray emission can be reconciled with the weak/absent optical Seyfert characteristics. The faintness of these objects in the X-ray, as well as in the optical domain, has not allowed us to study them in detail so far.

Near-infrared (NIR) imaging spectroscopy has considerable advantages over visible wavelengths: Besides the much smaller dust extinction, there are a number of NIR diagnostic lines (in emission and in absorption) to probe the excitation mechanisms and stellar content in galaxies. Among these are hydrogen recombination lines, rotational/vibrational transitions of $\mathrm{H}_{2}$, stellar features like the $\mathrm{CO}(2-0)$ and $\mathrm{CO}(6-3)$ absorption band heads, and forbidden lines like [Fe II] and [Si VI] (Hill et al. 1999; Mouri 1994; Marconi et al. 1994).

\subsection{Mrk 609}

Mrk 609 shows a mixture of nuclear star formation and Seyfert activity and appears to be a showcase for studying the nuclear activity with IFSs in order to probe the coeval existence of these phenomena.

There have been several observations of Mrk 609 (Figs. 1 and 16 for the SED). It is a member of the original sample of Osterbrock (1981), with which the role of reddening in Seyfert 1.8/1.9 galaxies was studied. These observations were followed up by X-ray, ultraviolet, optical, and infrared studies (Pappa et al. 2002; Rudy et al. 1988; Goodrich 1990; Rudy \& Rodriguez-Espinosa 1985), refining the classification of Mrk 609 as a starburst/Seyfert composite. The UV/optical line ratios and the X-ray spectrum furthermore indicate a small extinction towards the nucleus. Rudy et al. (1988) and Goodrich (1990) suggest that smaller-than-normal optical depths and lower ionization are responsible for the large Balmer decrement found in the optical spectrum (Fig. 15).

Optical $(\sim 0.6 \mu \mathrm{m}$; Nelson et al. 1996$)$ and NIR $(\sim 0.9 \mu \mathrm{m}$ Crenshaw et al. 2003) imaging observations of Mrk 609 with the Hubble Space Telescope (HST) have been used to address questions regarding the extension of the scattering medium in Seyfert 2 galaxies and the dependence of AGN fueling on the host morphology (Fig. 1). Nelson et al. studied a sample of Seyfert and non-Seyfert Markarian galaxies and found that Seyfert 1.8 and 1.9 show extended nuclear light profiles similar to Seyfert 2 galaxies. The energetics of the (circum-) nuclear region of Seyfert $2 \mathrm{~s}$ is strongly influenced by starburst activity. In combination with some scattering screen, this provides the extinction responsible for the observed shape of the light profiles. Seyfert 1.5 galaxies, on the other hand, have nuclear profiles typical of Seyfert 1 galaxies, which are unresolved and bright. Mrk 609 shows a strong Seyfert 1-like nucleus, but it appears to be slightly extended. Furthermore, weak broad H-recombination lines have been detected in optical spectra, which result in an intermediate Seyfert 1.5-1.8 classification (Goodrich 1990; Osterbrock 1981). This work supports the above findings. 

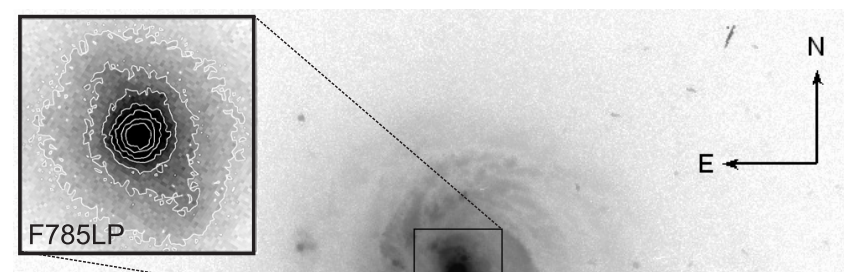

5 arcsec

F606W

Fig. 1. HST F606W $(\sim 0.6 \mu \mathrm{m})$ image of Mrk 609 (Malkan et al. 1998). The black box indicates the SINFONI field of view of $3 \times 3 \operatorname{arcsec}^{2}$. The upper left inset is an HST F785LP $(\sim 0.9 \mu \mathrm{m})$ image of the nuclear $3 \times 3 \operatorname{arcsec}^{2}$ (Nelson et al. 1996). Compare with Figs. 2d, 4a, and the structure image (Fig. 2) of Deo et al. (2006).

Crenshaw et al. and Deo et al. (2006) find a larger bar fraction for narrow-line Seyfert 1 (NLS1) galaxies than for broadline Seyferts (BLS1). NLS1 are believed to be systems accreting close-to or above the Eddington limit. The active state requires an efficient fuel supply, which can be supported by stellar bars. As a BLS1, the large-scale optical morphology of Mrk 609 appears flocculent and shows no signs of a bar (Crenshaw et al. 2003; Deo et al. 2006, Fig. 1). The nuclear morphology, however, deviates from a circular symmetric appearance. The NIR image of Nelson et al. (see inset in Fig. 1), which is less influenced by dust extinction than the optical image, reflects this situation. Two spiral arms turn out to connect to an elongated nuclear structure (northeast-southwest direction), probably resembling a nuclear stellar bar. In a recent numerical study, Englmaier \& Shlosman (2000) find that a grand-design nuclear two-arm spiral structure can be formed in the presence of a weak stellar bar. Only depending on the nuclear mass and the gas sound speed, such structures can allow for the inward mass transport beyond the inner Lindblad resonance towards radii of 50-100 pc. In respect thereof Deo et al. (2006) find that especially the class of flocculent spirals avoids the presence of large-scale bars, as appears to be the case for Mrk 609.

The paper is structured in the following manner. In Sect. 2 we describe the observations and data reduction. After a detailed analysis of the NIR and mm spectra, we discuss the results within this context of nuclear fueling in Sect. 3. In Sect. 4 we summarize the presented work and give an outlook. Unless otherwise noted, we use a cosmology with $H_{0}=70 \mathrm{~km} \mathrm{~s}^{-1} \mathrm{Mpc}^{-1}$, $\Omega_{\mathrm{m}}=0.3$, and $\Omega_{\Lambda}=0.7$ (Spergel et al. 2003) throughout this paper.

\section{Observations and data reduction}

\subsection{Near-infrared data}

The observations were carried out during the science verification phase $^{1}$ (October 2004) of SINFONI, the new AO-assisted integral field spectrometer mounted at Yepun, Unit Telescope 4 of the ESO Very Large Telescope in Chile (Eisenhauer et al. 2003).

\footnotetext{
${ }^{1}$ http://www.eso.org/science/vltsv/sinfonisv/ xrayagn.html
}

The AO guiding was carried out on the nucleus of Mrk 609. The average seeing was around 0.7 . The 100 mas pixel scale with a field-of-view (FOV) of $3^{\prime \prime} \times 3^{\prime \prime}$ was used. The 2D image on the sky was sliced by small mirrors into 32 slitlets, which then were reimaged onto a pseudoslit and dispersed onto a $2 \mathrm{k} \times 2 \mathrm{k}$ detector. The observations covered $J$ and $H+K$ bands with integration times of $5 \mathrm{~min}$ and $30 \mathrm{~min}$, respectively. Dispersion was achieved with the $J$ and $H+K$ gratings at a spectral resolution of $R_{J} \sim 2000$ and $R_{H+K} \sim 1500$. Successive target (T) and sky (S) observations (...TSST...) were acquired to produce sky-subtracted frames.

The reduction and reconstruction of the $3 \mathrm{D}$ cubes were carried out using the MPE reduction software spred 3.6 $6^{2}$, IDL, and QFitsView ${ }^{3}$. Bad pixel, cosmic rays, and flat field corrections were applied to the $2 \mathrm{D}$ raw frames. The $3 \mathrm{D}$ cubes were reconstructed using calibration frames for the slitlet distances and the light dispersion.

Intermediate standard-star observations (near in both time and airmass to the target exposures) of the G2V star HIP-021070 were used to correct for strong atmospheric (telluric) absorptions. They can be minimized by dividing the science target spectrum by the standard star spectrum. This step introduces features into the resulting spectrum that are intrinsic to the standard star and that can be accounted for by multiplication by the atmospheric-transmission-corrected solar spectrum. Since the standard star was saturated on the detector (see below in the context of flux calibration), we extracted the spectrum with telluric absorptions from the wings of the 2D image. The G2V spectral characteristics were removed by multiplying by the well known, high signal-to-noise solar spectrum (as provided by Maiolino et al. 1996). In the range of reduced atmospheric transmission (as indicated in Fig. 5), which is not covered by the Maiolino et al. spectrum, we interpolated the solar spectrum with a blackbody, because the original solar atlas did not show any intrinsic features in that range (Wallace et al. 1996).

Flux calibration is problematic, since the calibration-star observations saturated the detector. In order to approach a flux calibration, ignoring the fact of variability (up to 30\%) of Mrk 609 found by Rudy \& Rodriguez-Espinosa (1985), we used the 2MASS $J, H$, and $K$ s fluxes. We measured the fluxes within a $3^{\prime \prime}$ diameter aperture in the 2MASS atlas images and applied the fluxes to the $3^{\prime \prime} \times 3^{\prime \prime}$ SINFONI FOV. It has to be noted that this method has the drawback of ignoring the differences in spatial resolution. We expect an accuracy of the absolute flux to be around $40 \%$, but this is not of any significant importance, because we are primarily interested in measuring line ratios. For these, in principle, an absolute flux calibration is not necessary. We converted the magnitudes to flux densities using the Spitzer space telescope magnitude to flux density converter ${ }^{4}$. For $J$ we measured $J_{2 \text { MASS }}=13.68$, which corresponds to $f_{J 2 \mathrm{MASS}}=1.06 \times 10^{-14} \mathrm{~W} \mathrm{~m}^{-2} \mu \mathrm{m}^{-1}$ at $\lambda_{\mathrm{eff}}\left(J_{2 \mathrm{MASS}}\right)=1.235$. For the $H+K$ band we used the flux density at the effective wavelength of the $K$ s band. We measured $K \mathrm{~s}_{2 \mathrm{MASS}}=12.50$, which corresponds to $f_{K \text { s2MASS }}=4.29 \times 10^{-15} \mathrm{~W} \mathrm{~m}^{-2} \mu \mathrm{m}^{-1}$ at $\lambda_{\text {eff }}\left(K s_{2 M A S S}\right)=2.159$. The total flux within the SINFONI FOV was scaled according to these values. Because of the low value of $E(B-V)=0.056$ (Schlegel et al. 1998), no correction for Galactic extinction is applied.

\footnotetext{
${ }^{2}$ Kindly provided by Matthew Horrobin (MPE, Garching).

3 Written by Thomas Ott (MPE, Garching); http://www.mpe.mpg.de/ ott/QFitsView

${ }^{4}$ http://ssc.spitzer.caltech.edu/tools/magtojy/
} 

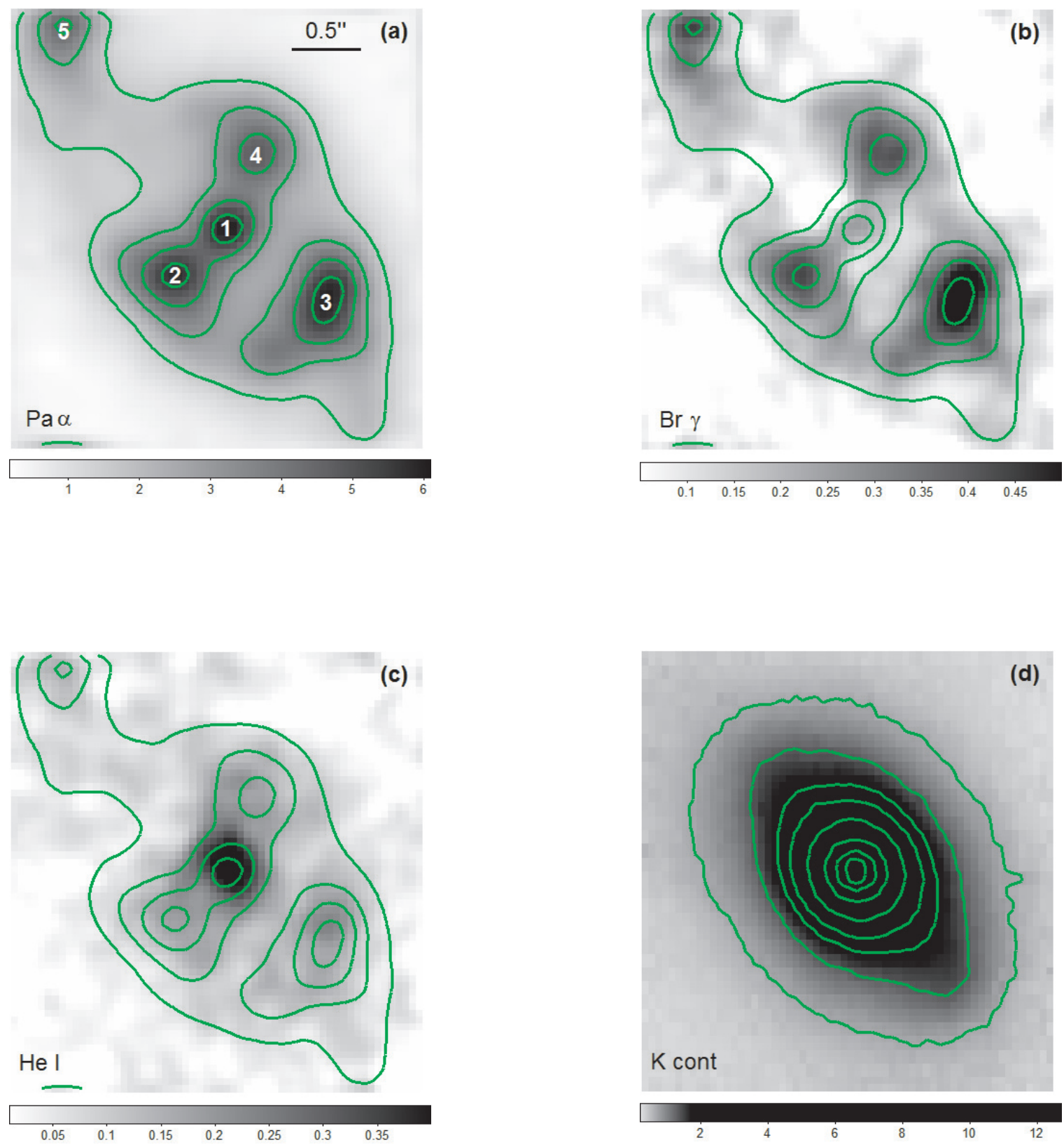

Fig. 2. $H+K$ emission line maps of recombination lines. They were created with QFITSVIEW by summing the flux over the emission line and subtracting the median value of the neighboring continuum (a)-c)). All images were smoothed with a 3-pixel Gaussian. The values of the color bars are in units of $10^{-19} \mathrm{~W} \mathrm{~m}^{-2}$. a) Narrow $\mathrm{Pa} \alpha$; contours are calculated relative to the peak flux $(90,70,50,30 \%)$. b) Bry overlayed with $\mathrm{Pa} \alpha$ contours. c) He I $2.059 \mu \mathrm{m}$ overlayed with Pa $\alpha$ contours. d) Continuum image in a region without emission lines around $2.2 \mu \mathrm{m}$. The panels are $3^{\prime \prime} \times 3^{\prime \prime}$ each. North is up and east is left. Regions are indicated in panel a) from which we extracted cummulative spectra. Regions 2 and 4 have a projected distance of about $420 \mathrm{pc}$, region $3 \approx 630 \mathrm{pc}$, and region $5 \approx 1.3 \mathrm{kpc}$ to the center.

From the final reconstructed 3D cube we extracted spectra and emission line maps. The emission line maps presented in Figs. 2-4 were created by summing up the flux of the emission line and then subtracting the average flux density of the continuum left and right of the line. The features seen in the $\mathrm{Pa} \alpha$ map define the regions where we extracted spectra (Figs. 5-8). The radius of the extraction regions is 5 pixels $(0 . ' 25)$.

\subsection{Millimeter data}

During an observing campaign of cluster galaxies with the Nobeyama $45 \mathrm{~m}$ telescope in March 2005 we additionally observed Mrk 609 in ${ }^{12} \mathrm{CO}(1-0)$. We used the autocorrelator as backend and an integration time of $45 \mathrm{~min}$. The measured antenna temperatures were transformed to a main-beam temperature using a main-beam efficiency of 0.38 . The baselinesubtracted $\mathrm{CO}(1-0)$ spectrum is presented in Fig. 13.

\section{Results and discussion}

In this section we describe the results of the spatially resolved spectroscopy of the circumnuclear environment of Mrk 609 and the $\mathrm{CO}(1-0)$ observation. 

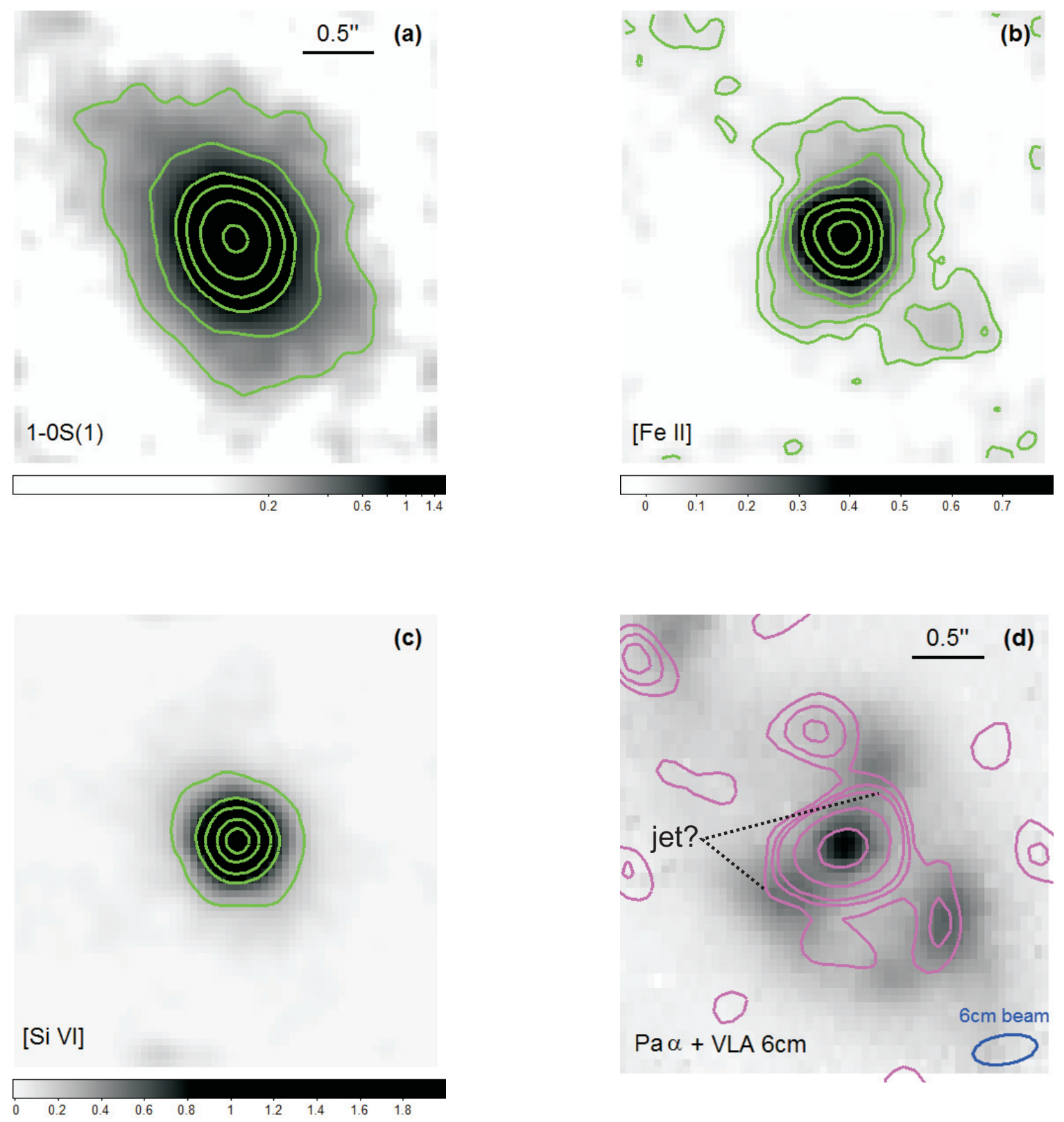

Fig. 3. $H+K$ emission line maps (using the same procedure as for Fig. 2) of: a) 1-0S(1) (contours: 90, 70, 50, 30, and 10\% of peak flux), b) $[\mathrm{Fe} \mathrm{II}] 1.664 \mu \mathrm{m}$ (contours: 90, 70, 50, 30, 20, 10, and 5\% of peak flux), and c) [Si VI] (contours: 90, 70, 50, 30, and 10\% of peak flux). The colorbar values are in units of $10^{-19} \mathrm{~W} \mathrm{~m}^{-2}$. Panel d) displays an overlay of the Pa $\alpha$ map from Fig. 2a with the $6 \mathrm{~cm}$ VLA map in (magenta) contours (from Ulvestad \& Wilson 1984). The blue ellipse in the lower right corner indicates the $6 \mathrm{~cm}$ beam size. The panels are $3^{\prime \prime} \times 3^{\prime \prime}$ each. North is up and east is left.

\subsection{Tracing the continuum and emission line gas}

The SINFONI imaging spectroscopy allows us to study the inner $2 \mathrm{kpc}$ of Mrk 609 at a spatial resolution of about $270 \mathrm{pc}$. We identified several emission and absorption features in the individual $J$ and $H+K$ spectra across the FOV (see Figs. 5 and 7). Among these are hydrogen recombination lines, rotational/vibrational lines of molecular hydrogen, forbidden transitions of [Fe II] and [Si VI], as well as stellar $\mathrm{CO}(6-3)$ and $\mathrm{CO}(2-0)$ absorption. From selected emission lines we produced continuum-subtracted line maps (Figs. 2-4).

In order to assess the spatial resolution we used the nuclear $\mathrm{Pa} \alpha$ emission. Fitting a 2D Gaussian to the nuclear $\mathrm{Pa} \alpha$ gives a rough estimate for the spatial resolution, because no other point-source observations were available for our observations. We found an $F W H M$ of about 8 pixel $\left.(\sim 0)^{\prime} 4\right)$ for the minor axis, while the major axis is clearly extended. We expect this to be a reasonable measure of the point-spread function at all wavelengths. This size corresponds to a linear scale of about $270 \mathrm{pc}$ $\left(1^{\prime \prime} \approx 680 \mathrm{pc}\right)$ at the redshift of $z=0.0345$.

The morphologies of the hydrogen recombination line maps cleary differ from the continuum maps. The $\mathrm{Pa} \alpha$ map reveals five emission peaks that are indicated in Fig. 2a. The continuum emission presented in panel (d) of Fig. 2 shows an elliptical structure extending in a northeast/southwest direction and connecting to the large-scale spiral arms visible in the HST image (Fig. 1). This morphology is similar to that of the nuclear stellar bars found in several other galaxies (e.g. Erwin \& Sparke 2002; 


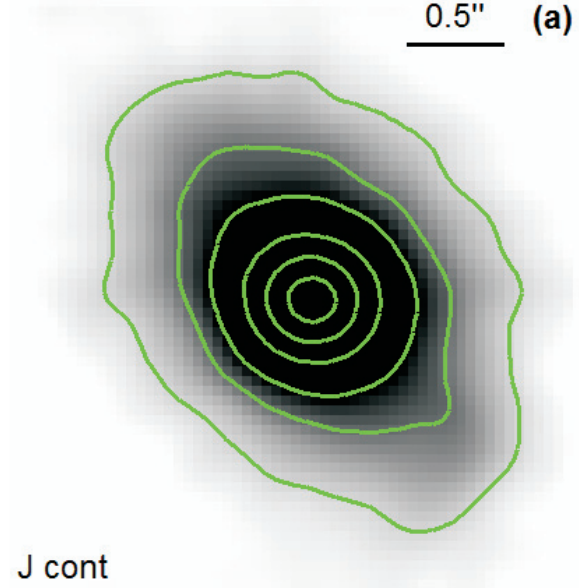

\begin{tabular}{lllllll}
\hline 1 & 2 & 3 & 4 & 5 & 6 & 7
\end{tabular}

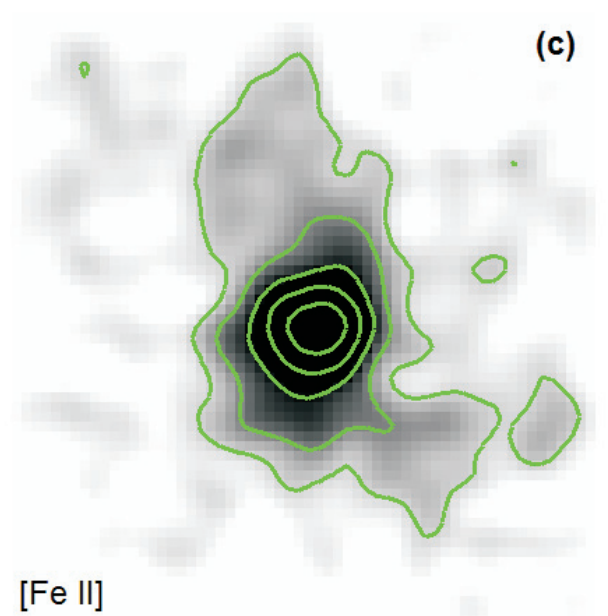

(c)

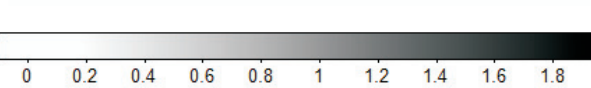

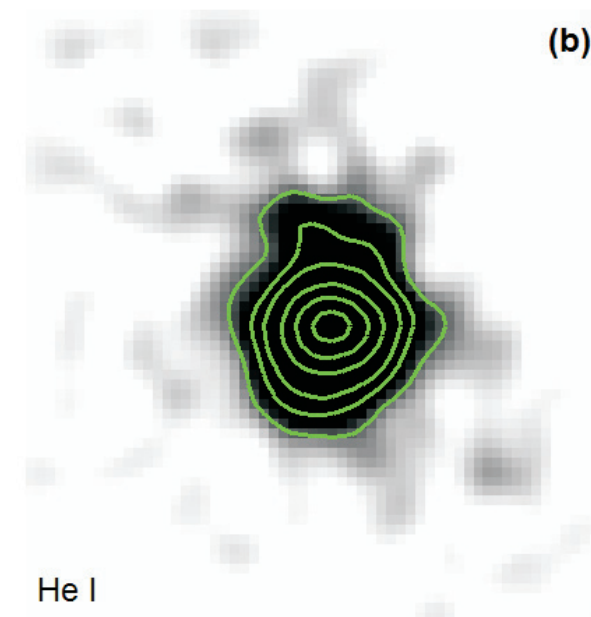

(b)
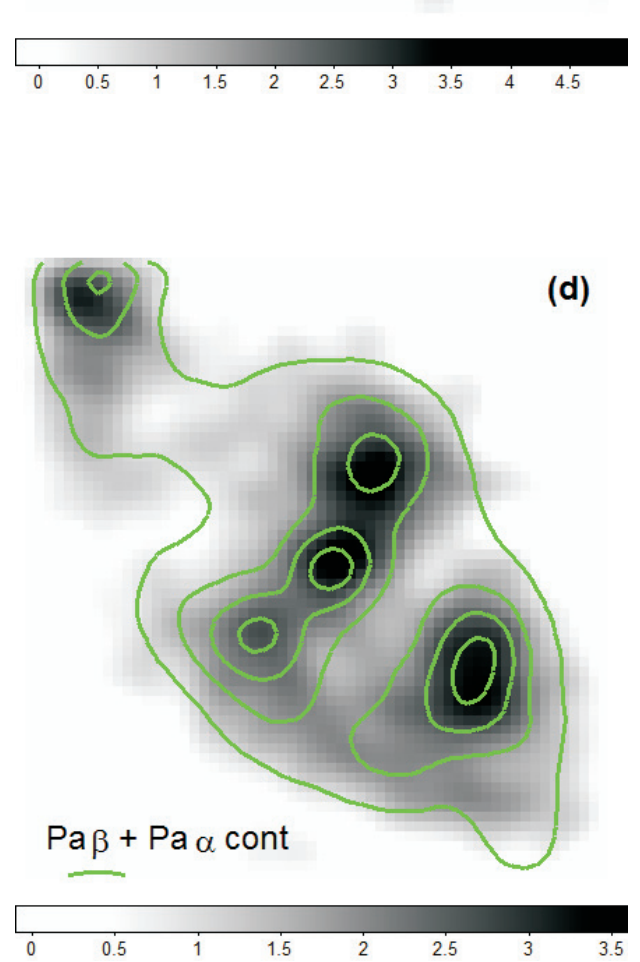

Fig. 4. $J$-band continuum and emission line maps of: a) $1.25 \mu$ m continuum (contours: $90,70,50,30$, 20, and $10 \%$ of peak flux), b) He I $1.083 \mu \mathrm{m}$ (contours: 90, 70, 50, 30, 20, and 10\% of peak flux), c) Fe II $1.257 \mu$ m (contours: 90, 70, 50, 30, and 15\% of peak flux), and d) Pa $\beta$ overlayed with $\mathrm{Pa} \alpha$ in contours (see Fig. 2a). Color bar values are in units of $10^{-19} \mathrm{~W} \mathrm{~m}^{-2}$. The panels are $3 \times 3 \operatorname{arcsec}^{2}$ each. North is up and east is left.

Martini et al. 2003). Results of $N$-body simulations carried out by Patsis (2005), in which stellar particles move on (quasi-) periodic orbits in a bar potential, also resemble the nuclear continuum morphology of Mrk 609.

The $\mathrm{Pa} \alpha$ peaks are apparently aligned with the barred continuum. One peak is centered on the nucleus. The four other peaks lie at the tip of the major axis where the bar connects to the spiral arms (regions 3 and 5) and on the minor axis (regions 2 and 4). In addition to the clumpy $\mathrm{Pa} \alpha$ emission a fainter and smoother $\mathrm{Pa} \alpha$ component is visible, which extends between the distinct regions. There is a striking similarity between this configuration and the observation of Emsellem et al. (2003), who detected a nuclear two-arm gas spiral in the early type galaxy NGC 2974. This spiral structure ( $\sim 200 \mathrm{pc}$ radius) is embedded in a $\sim 500 \mathrm{pc}$ radius nuclear stellar bar. These dimensions are roughly the same (within a factor of 2) as for Mrk 609 (Fig. 2a and d). Simulations show that gaseous material can be transported along these spiral arms towards the central region (Englmaier \& Shlosman 2000). Mrk 609 has also been studied at $6 \mathrm{~cm}$ wavelengths (Ulvestad \& Wilson 1984). Figure 3d displays the $\mathrm{Pa} \alpha$ map overlayed with the radio contours. The radio emission appears to be slightly resolved and it also coincides with the recombination line emission. Accordingly, the $6 \mathrm{~cm}$ radio continuum traces the zones of ongoing star formation. As indicated in Fig. 2d, there is an extension towards regions 2 and 4, which could also be interpreted as a jet component. Regions 2 and 4, therefore, might be excited by the radio jet impinging on the inter-stellar medium. In this case the nuclear spiral scenario has to be altered. See Sects. 3.2.1 and 3.5 for further discussion.

Next, we extracted 1D spectra from the $H+K$ and $J$ bands for each of the five regions by summing up the individual spectra within apertures of 5 pixel radius, centered on the $\mathrm{Pa} \alpha$ emission peaks (see Figs. 5 and 7). The apertures sizes have been chosen to represent the angular resolution of the observation. In the 


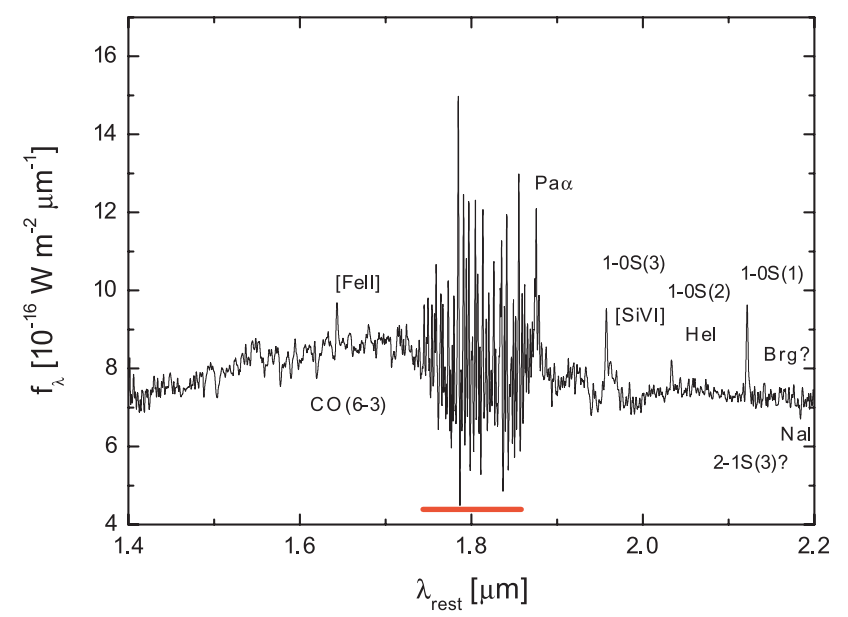

Fig. 5. $H+K$ rest-frame spectrum extracted from a 5-pixel radius aperture centered on the nucleus (region 1 in Fig. 2a). Prominent lines are indicated (cf. Table 1). The high noise around $1.8 \mu \mathrm{m}$, indicated by the thick bar, is due to reduced atmospheric transmission between the $H$ and the $K$ bands.

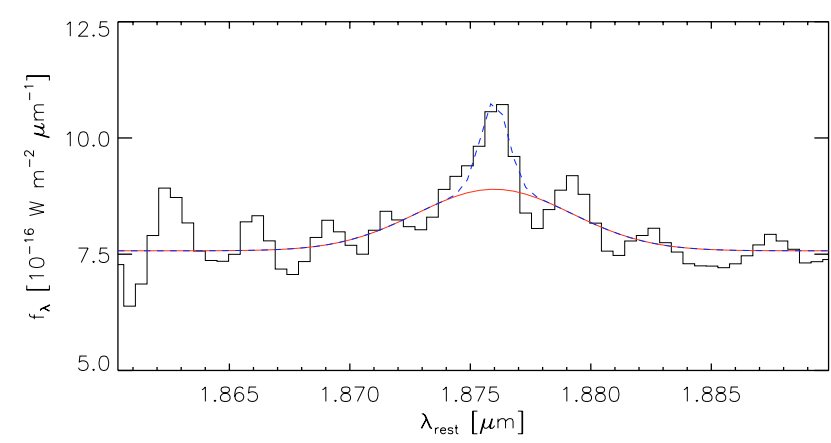

Fig. 6. Rest-frame spectrum around $\mathrm{Pa} \alpha$, extracted from the nuclear 5 pixel radius aperture. The spectrum has been smoothed with a 3-pixel boxcar. The blue dashed and red solid lines represent Gaussian fits to the narrow and broad components of $\mathrm{Pa} \alpha$.

resulting spectra we measured line fluxes for individual lines by direct measurement. Line fluxes and $F W H M$ of the line profiles are given in Table 1. The upper part of the table corresponds to the $J$ part of the spectrum, the lower one to the $H+K$ part. Fluxes are given in units of $10^{-19} \mathrm{~W} \mathrm{~m}^{-2}$ and observed line widths are given in $\mathrm{km} \mathrm{s}^{-1}$. Errors of the line fluxes were estimated from the noise of the neighboring continuum alone. One has to keep in mind that errors introduced by the flux calibration become relevant when comparing line fluxes between bands $(J$ and $H+K)$ or when using absolute fluxes (see discussion about calibration in Sect. 2.1). The measurement of the FWHM depends on the shape of the continuum. We estimate its error to be on the order of $30 \%$. In the case of $\mathrm{Pa} \alpha$ and $\mathrm{Pa} \beta$ we fitted a broad and narrow Gaussian component with a common line center (Figs. 6 and 8). The measurement of the broad components is quite intricate, since these lines are located in problematic regions of the spectrum. For example, $\mathrm{Pa} \alpha$ is located at the red end of the atmospheric transmission minimum $(\sim 1.8 \mu \mathrm{m})$, and the spectrum is very noisy around this feature. As for $\mathrm{Pa} \beta$ and for $\mathrm{He} \mathrm{I}$, they are located at the beginning and at the end of the $J$-band spectrum and are also influenced by increased noise. For the nucelar 1-0S(3)/[Si VI] complex, we fitted two Gaussians and a telluric absorption component. A fit without the telluric component recovers the 1-0S(3) flux and linewidth within the errors given in Table 1 . In the case of the [Si VI], the flux and linewidth are

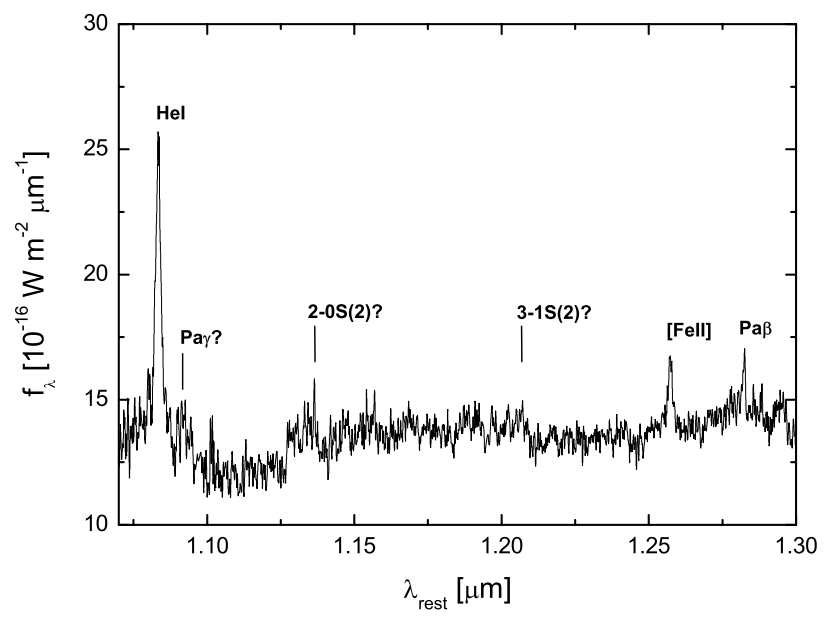

Fig. 7. $J$ rest-frame spectrum extracted from a 5 pixel radius aperture centered on the nucleus (region 1 in Fig. 2a). Prominent lines are indicated (cf. Table 1).

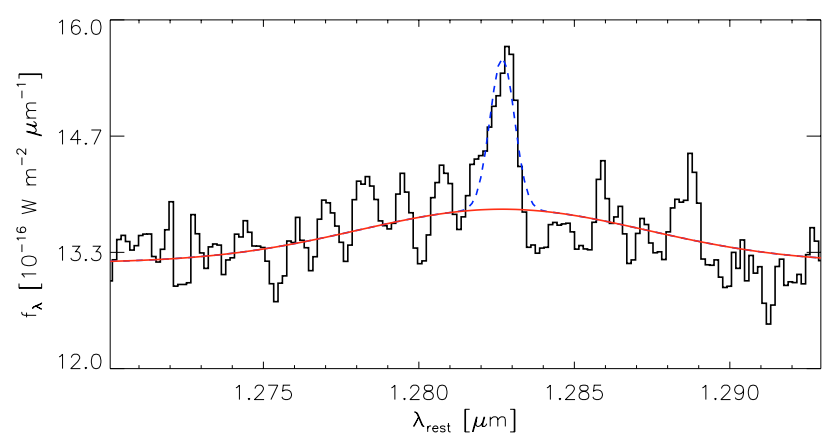

Fig. 8. Rest frame spectrum around $\mathrm{Pa} \beta$, extracted from the central 5 pixel radius aperture. The spectrum has been smoothed with a 3-pixel boxcar and the result of the fit of a narrow (blue dashed curve) and a broad (red solid curve) Gaussian component with a common line center is shown.

considerably reduced (by about $60 \%$ and $30 \%$, respectively). Thus, primarily the properties of $[\mathrm{Si} \mathrm{VI}]$ and not of $1-0 \mathrm{~S}(3)$ are influenced. Since we only state the presence of the former as an indicator of nuclear activity and do not consider it any further, we neglect the telluric absorption for the 1-0S(3) measurement.

In contrast to $\mathrm{H} \alpha$ and $\mathrm{H} \beta$ at visible wavelengths (Fig. 15), $\mathrm{Pa} \alpha$ (Fig. 6) and $\mathrm{Pa} \beta$ (Fig. 8) clearly show a broad component with an FWHM of about $3000 \mathrm{~km} \mathrm{~s}^{-1}$ (after correcting for the instrumental spectral resolution). This supports the Seyfert classification of Mrk 609 as deduced from the X-ray properties (Pappa et al. 2002). In the $J$-band spectrum, there is also evidence of a broad He I $1.083 \mu \mathrm{m}$ component, but the low signal-to-noise ratio prevents us from decomposing broad and narrow components. Therefore, we only state the flux from direct measurement of the narrow component in Table 1 . The broad component also agrees with the line width of the broad $\operatorname{Ly} \alpha\left(\sim 3600 \mathrm{~km} \mathrm{~s}^{-1}\right.$, but blended with $\mathrm{N} \mathrm{V}$ ) found by Rudy et al. (1988), when taking the International Ultraviolet Explorer (IUE) spectral resolution of $7 \AA$ into account. The coronal [Si VI] emission visible in the nuclear spectrum (Figs. 5 and 3c for the line map) is another common feature of the extreme energetics in AGN (Marconi et al. 1994; Prieto et al. 2005).

Using the unreddened case-B line ratio of $\mathrm{Pa} \alpha / \mathrm{H} \beta=0.332$ (Osterbrock 1989), we find that the broad component should be detectable in the visible; but as mentioned in the introduction, Mrk 609 exhibits unusual line ratios. Intrinsic extinction can be 
Table 1. Emission line fluxes for the 5 regions identified in the Pa $\alpha$ line map (Fig. 2a).

\begin{tabular}{|c|c|c|c|c|c|c|c|c|c|c|}
\hline \multirow{2}{*}{$\begin{array}{c}\text { Line } \\
\lambda[\mu \mathrm{m}]\end{array}$} & \multicolumn{2}{|c|}{ Region 1} & \multicolumn{2}{|c|}{ Region 2} & \multicolumn{2}{|c|}{ Region 3} & \multicolumn{2}{|c|}{$\overline{\text { Region } 4}$} & \multicolumn{2}{|c|}{ Region 5} \\
\hline & Flux & $F W H M$ & Flux & $F W H M$ & Flux & $F W H M$ & Flux & $F W H M$ & Flux & $F W H M$ \\
\hline He I $\lambda 1.0833$ narrow & $31.8 \pm 10.6$ & 540 & - & - & - & - & - & - & - & - \\
\hline$[\mathrm{Fe}$ II $] \lambda 1.257$ & $5.1 \pm 1.0$ & 439 & $1.3 \pm 0.3$ & 327 & $0.7 \pm 0.1$ & 160 & $1.3 \pm 0.2$ & 303 & - & - \\
\hline $\mathrm{Pa} \beta$ narrow $^{a}$ & $1.9 \pm 0.6$ & 214 & $1.7 \pm 0.1$ & 150 & $2.2 \pm 0.1$ & 163 & $2.2 \pm 0.2$ & 183 & - & - \\
\hline $\mathrm{Pa} \beta$ broad $^{a}$ & $15.0 \pm 7.0$ & 3400 & - & - & - & - & - & - & - & - \\
\hline$[\mathrm{Fe} \mathrm{II}] \lambda 1.644^{c}$ & $2.7 \pm 0.2$ & 410 & $0.6 \pm 0.1$ & 240 & $0.3 \pm 0.1$ & 200 & $0.5 \pm 0.1$ & 260 & $0.07 \pm 0.01$ & 200 \\
\hline $\mathrm{Pa} \alpha$ narrow & $5.3 \pm 0.6$ & 300 & $7.9 \pm 0.2$ & 270 & $7.0 \pm 0.1$ & 200 & $5.4 \pm 0.2$ & 200 & $0.22 \pm 0.01$ & 210 \\
\hline $\mathrm{Pa} \alpha$ broad & $16.4 \pm 5.6$ & 3000 & - & - & - & - & - & - & - & - \\
\hline $1-0 \mathrm{~S}(3)^{b, c}$ & $5.0 \pm 0.8$ & 317 & $1.1 \pm 0.2$ & 371 & $0.5 \pm 0.1$ & 345 & $0.6 \pm 0.2$ & 471 & $0.05 \pm 0.02$ & 230 \\
\hline$[\mathrm{Si} \mathrm{VI}]^{b, c}$ & $16.4 \pm 4.2$ & 2130 & - & - & - & - & - & - & - & - \\
\hline $1-0 \mathrm{~S}(2)$ & $2.1 \pm 0.4$ & 308 & $0.6 \pm 0.1$ & 366 & $0.20 \pm 0.06$ & 321 & $0.4 \pm 0.1$ & 333 & $0.06 \pm 0.02$ & 450 \\
\hline He I $\lambda 2.058$ & $0.3^{d}$ & 300 & $0.3 \pm 0.1$ & 338 & $0.20 \pm 0.05$ & 200 & $0.20 \pm 0.05$ & 200 & $0.07 \pm 0.01$ & 200 \\
\hline $2-1 S(3)^{e}$ & 0.2 & 317 & 0.1 & 322 & 0.1 & 312 & 0.1 & 316 & 0.02 & 300 \\
\hline $1-0 \mathrm{~S}(1)$ & $6.4 \pm 0.4$ & 330 & $1.6 \pm 0.1$ & 375 & $0.6 \pm 0.04$ & 270 & $0.9 \pm 0.1$ & 300 & $0.10 \pm 0.01$ & 300 \\
\hline $\mathrm{Br} \gamma$ & $0.37^{d}$ & 300 & $0.5 \pm 0.1$ & 267 & $0.6 \pm 0.1$ & 200 & $0.4 \pm 0.1$ & 200 & $0.18 \pm 0.01$ & 200 \\
\hline
\end{tabular}

${ }^{a}$ Decomposition using two Gaussian components. ${ }^{b} 1-0 \mathrm{~S}(3)$ and [Si VI] are decomposed using two Gaussian components. ${ }^{c}$ Result strongly influenced by telluric absorption. ${ }^{d}$ Upper limit, assuming the $F W H M$ of the narrow Pa $\alpha$ component. ${ }^{e}$ Upper limit, assuming the average $F W H M$ of the other molecular hydrogen lines.

probed by comparing the ratios of hydrogen recombination lines with case-B values from Osterbrock (1989). For $T=10^{4} \mathrm{~K}$, $n_{\mathrm{e}}=10^{4} \mathrm{~cm}^{-3}$, and no extinction, we expect $\mathrm{Pa} \alpha / \mathrm{Br} \gamma=12.4$. Table 2 lists this ratio among others for the regions discussed here. Within the measurement uncertainties, the $\mathrm{Pa} \alpha / \mathrm{Br} \gamma$ ratios are consistent with no intrinsic extinction. There is in fact a trend of the ratios being a bit larger then the case-B value. This is especially the case for the nuclear region, where the corresponding ratios of the broad components also show the same behavior. This result agrees with low $\mathrm{H}$ column densities derived from the X-ray spectra (Pappa et al. 2002) and the unusual line ratios found in the UV/optical by Rudy et al. (1988), who explained these ratios with low optical depths and low ionization parameters.

The $1.66 \mu \mathrm{m} / 2.16 \mu \mathrm{m}$ continuum color map (Fig. 11) is sensitive to reddening caused by warm dust. The map shows reddening along the major bar axis, with peaks at the nucleus and at the tip of the bar, meeting the spiral arms. Previous studies of the NIR continuum of Seyfert galaxies concluded that their $J-H$ and $H-K$ nuclear colors can be explained by a mixture of a stellar component and a warm/hot dust component in emission (e.g. Hyland \& Allen 1982; Glass \& Moorwood 1985; Kotilainen et al. 1992; Alonso-Herrero et al. 1996). Using the $H$ and $K$ zero points from Table 3.4 in Glass (1999), the $H-K$ colors range from 0.6 in the outer regions up to 1.0 at the nucleus. These are compatible with warm $(\sim 500 \mathrm{~K})$ dust emission and small extinction (cf. Glass \& Moorwood 1985). As Calzetti et al. (1996) and Fischera et al. (2003) furthermore show, the effects of dust in starbursts can often be fairly well modelled by a homogeneous or clumpy/turbulent foreground screen. Since the extinction curve is very flat in the NIR (Draine 1989) and since the wavelengths of the lines used in the present analysis have small separations, the differential reddening between these lines will be small. Therefore, the $H-K$ reddening will only have a neglegible influence on the measured line ratios.

Due to the uncertainties in the absolute calibration, where we do not have reliable knowledge of the host galaxy and nuclear contribution in the measuring aperture, we will not use the $\mathrm{Pa} \beta / \mathrm{Pa} \beta$ ratio as an indicator for extinction. Also the $J$ band spectrum shows no significant extinction. If we assume the same case-B conditions as above, we would expect not to detect the Pay line (close to He I $1.0883 \mu \mathrm{m}$ ). Figure 4, however, shows
Table 2. Ratios of prominent emission lines using the narrow components.

\begin{tabular}{cccc}
\hline \hline Region & $\frac{\mathrm{Pa} \alpha}{\mathrm{Br} \gamma}$ & $\frac{\mathrm{H}_{2} 2.121 \mu \mathrm{m}}{\mathrm{Br} \gamma}$ & $\frac{[\mathrm{Fe} \mathrm{II}] 1.257 \mu \mathrm{m}}{\mathrm{Pa} \beta}$ \\
\hline 1 & $>14.3(>13.7)^{a}$ & $>17.3$ & $3 \pm 1$ \\
2 & $15 \pm 3$ & $3.2 \pm 0.7$ & $0.8 \pm 0.2$ \\
3 & $12 \pm 2$ & $1.0 \pm 0.2$ & $0.32 \pm 0.04$ \\
4 & $14 \pm 3$ & $2.3 \pm 0.6$ & $0.5 \pm 0.1$ \\
5 & $12.2 \pm 0.1$ & $0.6 \pm 0.2$ & - \\
\hline
\end{tabular}

${ }^{a}$ Broad component in brackets.

faint signs of $\mathrm{Pa} \gamma$, which points towards the same conclusion of small extinction around the nucleus.

$\mathrm{Pa} \beta, \mathrm{Br} \gamma$, and $\mathrm{He} \mathrm{I} 2.058 \mu \mathrm{m}$ exhibit the same spatial distribution (Figs. 2b, c, and Fig. 4d), and their intrinsic line widths are comparable and are spectroscopically unresolved. This indicates that the lines arise in the same parcels of gas. Note that on the nucleus $\mathrm{Br} \gamma$ is only measured as an upper limit and He I $2.058 \mu \mathrm{m}$ only has a narrow component. On the other hand He I $1.0883 \mu \mathrm{m}$ is just concentrated on the nucleus (Fig. 4b) and has both a broad component and a narrow one.

\subsection{Molecular hydrogen and iron}

The $\mathrm{H}_{2}$ emission lines originate in surfaces of molecular clouds exposed to stellar or nuclear radiation. The observed line strengths and ratios strongly depend on the excitation mechanisms of $\mathrm{H}_{2}$ discussed below.

[Fe II] emission is believed to originate in partially ionized zones, which occur around supernovae or the active nucleus. Such regions can be produced by the hard ionizing X-ray/UV continuum or by shocks in the circumnuclear gas or in supernova remnants (e.g. Alonso-Herrero et al. 1997).

\subsubsection{Kinematics of the $\mathrm{H}_{2}$ and [FeII] lines}

The $H+K$ spectra exhibit a couple of rotational/vibrational lines of molecular hydrogen (Fig. 5). The most prominent is the $1-0 S(1)$ transition. Figure $3 b$ shows the spatial distribution of $\mathrm{H}_{2}$, which appears rather confined to the nucleus. Most of the 
$1-0 \mathrm{~S}(1)$ emission is concentrated within 260 pc (30\% peak contour in Fig. 3a) around the nucleus. The emission further shows a smooth decline towards the outer regions as it follows the continuum contours. This is in agreement with previous investigations of the molecular content of samples of Seyfert galaxies, which showed that the gas is concentrated in a disk around the centers of the galaxies (Reunanen et al. 2003; Rodríguez-Ardila et al. 2005). The [Fe II] emission is oriented almost perpendicular to the $\mathrm{H}_{2}$ emission and follows the recombination line gas (Figs. $3 b$ and $2 \mathrm{a}-\mathrm{c}$ ). Comparison of the line width of $\mathrm{H}_{2}$ with the one of the narrow lines can thus yield constraints on the location of the molecular and narrow-line gas. We consider line widths to be spectroscopically-resolved, if the FWHM is larger than $280 \mathrm{~km} \mathrm{~s}^{-1}$, i.e. $\sqrt{2}$ times the spectral resolution of $200 \mathrm{~km} \mathrm{~s}^{-1}$. From Table 1 we find that the molecular hydrogen emission is slightly resolved spectroscopically and that the line widths are similar in all regions. The $\mathrm{H}_{2}$ lines are also always broader than the narrow recombination lines. In contrast to this, [Fe II] $1.257 \mu \mathrm{m}$ appears to be resolved spectroscopically only on the nucleus and in regions 2 and 4. In these three regions [Fe II] is also broader than the narrow recombination lines and even broader than molecular hydrogen. Apart from that its FHWM is similar to that of the narrow recombination lines. Off-nuclear [Fe II] follows the patchy $\mathrm{Pa} \alpha$ emission/ $\mathrm{Br} \gamma / \mathrm{He}$ I distribution, thereby tracing star formation. On the other hand, the unusual broadening of the iron emission in regions 1, 2, and 4 might also be related to a jet-like extension (southeast-northwest direction) in the $6 \mathrm{~cm}$ VLA continuum map of Ulvestad \& Wilson (1984). Figure $3 \mathrm{~d}$ displays our $\mathrm{Pa} \alpha$ map overlayed with the radio map in contours. It is rather speculative but conceivable that a radio jet might alter the iron gas kinematics (cf. discussion in Reunanen et al. 2003).

These observations seem to confirm previous results by Reunanen et al. (2003) and Rodríguez-Ardila et al. (2005) that the $\mathrm{H}_{2}$ kinematics is decoupled from the [Fe II] and narrow recombination line kinematics.

However, because of the combination of low inclination and limited spectral resolution, we cannot detect any considerable dynamics from line shifts.

\subsection{2. $\mathrm{H}_{2}$ excitation mechanism by line ratios}

Three distinct processes are commonly discussed: (1) UV fluorescence (non-thermal); (2) X-ray heating (thermal); and (3) shocks (thermal). The processes result in different $\mathrm{H}_{2}$ responses and can thus helping distinguishing the dominant excitation process (e.g. Mouri 1994).

1. Excitation via $U V$-fluorescence can occur via UV pumping and/or collision with $\mathrm{H}$-atoms, which are controlled by temperature, density, and strength of the UV radiation field. Within a warm, high-density gas in a strong UV radiation field, thermal line ratios are found for the lower vibrational levels. In this case the lower levels are dominated by collisional excitation, whereas the higher levels are populated via UV pumping. To distinguish this scenario from pure shock excitation, observations of the high transitions are essential. In the UV excited gas, strong $2-1 \mathrm{H}_{2}$ lines are expected, as well as strong lines in the $H$ band, e.g. $6-4 \mathrm{Q}(1) 1.6 \mu \mathrm{m}$ or 1-0S(7) $1.75 \mu \mathrm{m}$ (cf. Table 2 of Black \& van Dishoeck 1987). The $H$-band lines only become weak relative to the $K$-band lines in the case of very high densities and high UV intensities. Since we detected no $\mathrm{H}_{2}$ lines in the $H$ band and the
Table 3. Emergent $\mathrm{H}_{2}$ and [Fe II] fluxes using the models of Maloney et al. (1996).

\begin{tabular}{ccccc}
\hline \hline & \multicolumn{2}{c}{$n=10^{5} \mathrm{~cm}^{-3}$} & \multicolumn{2}{c}{$n=10^{3} \mathrm{~cm}^{-3}$} \\
$d$ & $1-0 \mathrm{~S}(1)$ & {$[\mathrm{Fe}$ II $]$} & $1-0 \mathrm{~S}(1)$ & {$[\mathrm{Fe}$ II $]$} \\
\hline 50 & 1450.0 & 0.5 & - & - \\
80 & 15.0 & 0.0 & 460.0 & 145.0 \\
420 & 15.0 & 0.0 & 0.05 & 0.0 \\
630 & 9.0 & 0.0 & 0.08 & 0.0 \\
1300 & 3.0 & 0.0 & 0.15 & 0.0 \\
\hline
\end{tabular}

2-1 $\mathrm{H}_{2}$ lines are only measured as upper limits, UV radiation as the main excitation mechanism seems unlikely.

2. Excitation by X-rays or cosmic rays. Tine et al. (1997), Draine \& Woods (1990), as well as Maloney et al. (1996), analyzed the excitation of $\mathrm{H}_{2}$ by $\mathrm{X}$-rays and cosmic rays. Obvious non-thermal line spectra from these X-raydominated regions (XDRs) are only observable, if the temperature is well below $1000 \mathrm{~K}$ and the ionization-rate per $\mathrm{H}$-atom is below $10^{-15} \mathrm{~cm}^{3} \mathrm{~s}^{-1}$. At higher temperatures, the lower vibrational levels will be populated mainly by collisions, whereas higher ionization-rates will destroy the $\mathrm{H}_{2}$ molecules. Lepp \& McCray (1983) derived the relation $L_{\mathrm{IR}} \approx 10^{-3} L_{\mathrm{X}}$ between the $1-10 \mathrm{keV} \mathrm{X}$-ray luminosity and the 1-0S(1) line luminosity for isobaric models containing a compact X-ray source. The 2-10 keV X-ray flux of Mrk 609 (Pappa et al. 2002) is about $2.7 \times 10^{-15} \mathrm{~W} \mathrm{~m}^{-2}$ and translates to a $1-0 \mathrm{~S}(1)$ line flux of about $\sim 10^{-18} \mathrm{~W} \mathrm{~m}^{-2}$. This is comparable in magnitude to the measured 1-0S(1) flux, if summing over all regions. The analysis of the line ratios, however, does not strongly support excitation via X-rays.

3. Thermal excitation via shock fronts. Thermal excitation occurs through collisions with $\mathrm{H}$ or $\mathrm{H}_{2}$. The population of the electronic ground levels in the ro-vibrational transitions represents a Boltzmann distribution. Then the temperature of the gas represents a kinetic temperature that could be as high as $2000 \mathrm{~K}$ or more. See for example Draine \& McKee (1993) for a review.

In order to test whether the [Fe II] and $\mathrm{H}_{2}$ emission can be caused by X-ray heating, we used the models of Maloney et al. (1996) to estimate the emergent $1-0 \mathrm{~S}(1)$ and [Fe II] fluxes of a gas cloud illuminated by a source of hard X-rays with an intrinsic luminosity $L_{\mathrm{X}}$. The cloud is at a distance $d$ from the X-ray source and has an electron density $n_{\mathrm{e}}$. The cooling is then given by the effective ionization parameter $\xi_{\text {eff }}$ :

$\xi_{\text {eff }}=1.26 \times 10^{-4} \frac{f_{\mathrm{X}}}{n_{5} N_{22}^{0.9}}$

where $f_{\mathrm{X}}$ is the incident hard X-ray flux at the distance $d$ [pc] from the X-ray source, $n_{5}\left[10^{-5} \mathrm{~cm}^{-3}\right]$ is the total hydrogen gas density, and $N_{22}\left[10^{22} \mathrm{~cm}^{2}\right]$ the AGN intrinsic attenuating column density. The value of $f_{\mathrm{X}}$ can be calculated via $L_{\mathrm{X}} / 4 \pi d^{2}$. Maloney et al. (1996) calculate the emergent intensities for two gas densities, $10^{5} \mathrm{~cm}^{-3}$ and $10^{3} \mathrm{~cm}^{-3}$, which can be directly read off their Fig. 6a,b. Then, the flux can be calculated using the solid angle of the cloud region, i.e. the aperture of a 10-pixel diameter $\left(0 . ' 5\right.$ corresponding to $\left.4.6 \times 10^{-12} \mathrm{sr}\right)$. The BeppoSAX $0.1-10 \mathrm{keV} X$-ray luminosity is $6.3 \times 10^{42} \mathrm{erg} \mathrm{s}^{-1}$ (in the high state) and the absorbing column density is $N_{\mathrm{H}} \leq 1.32 \times 10^{21} \mathrm{~cm}^{-2}$ for a single power-law model (Pappa et al. 2002). For the indicidual regions, Table 3 lists the $\mathrm{H}_{2}$ and [FeII] fluxes in units of $10^{-20} \mathrm{~W} \mathrm{~m}^{-2}$ for the distances $d$ in pc. 


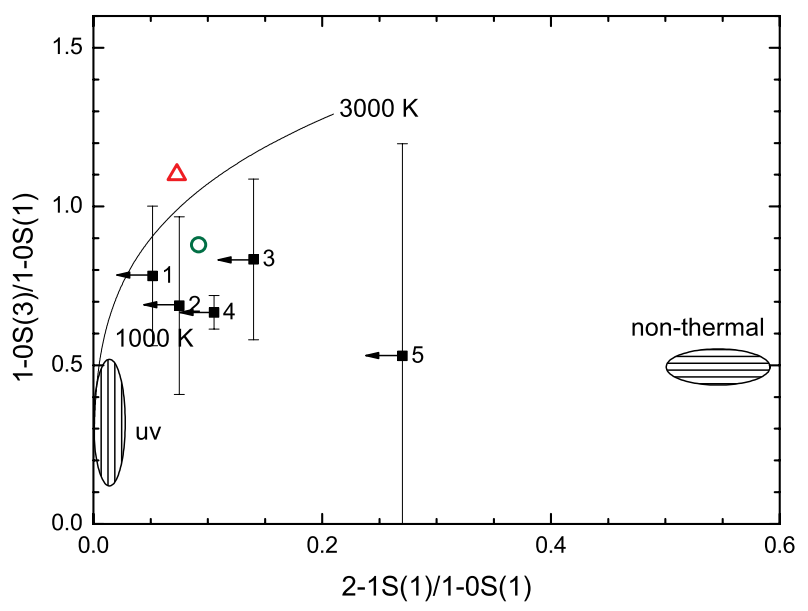

Fig. 9. $\mathrm{H}_{2}$ diagnostics diagram using the two line ratios $2-1 \mathrm{~S}(1) / 1-0 \mathrm{~S}(1)$ vs. $1-0 \mathrm{~S}(3) / 1-0 \mathrm{~S}(1)$. The region with the horizontal fill pattern covers the non-thermal models of Black \& van Dishoeck (1987). The vertically patterned region are the thermally uv-excited models of Sternberg \& Dalgarno (1989). The open triangle corresponds to the X-ray heating models of Draine \& Woods (1990) and the open circle to the shock model of Brand et al. (1989). Filled squares are the measured line ratios with numbers indicating the extraction region (see Fig. 2a).

Taking the uncertainties in our flux calibration into account, the $\mathrm{H}_{2}$ estimates show that X-ray heating can account for part of the observed 1-0S(1) flux (cf. Table 1) in the nuclear region. The model also predicts the $2-1 \mathrm{~S}(1)$ and the $\mathrm{Br} \gamma$ fluxes. The expected line ratios approximately resemble those of the observed 2-1S(1)/1-0S(1) and 1-0S(1)/Br $\gamma$ ratios. On the other hand, [Fe II] $1.644 \mu \mathrm{m}$ appears not to be excited by X-ray heating, except for the nuclear region in case of the lower electron density environment. This indicates that other processes like shock heating or fluorescence have to be accounted for. In fact, the NIR diagnostic diagram (Fig. 14) points towards the importance of shock heating.

In summary, the observed line ratios displayed in Fig. 9 agree quite well with those of a shock model with $2000 \mathrm{~K}$ from Brand et al. (1989) for all regions. A scenario with mixed excitation is possible and more realistic as well. Fitting the 2-1 S(1)/1-0 S(1) ratio with the models 14 (UV excitation) and S2 (shock excitation) of Black \& van Dishoeck (1987) for regions $1-4$ about $96 \%$ of the line emission should be due to thermal excitation and about $4 \%$ due to UV pumping. For region 5 thermal excitation could account for up to $60 \%$ and UV pumping for up to $40 \%$.

\subsubsection{The $\mathrm{H}_{2}$ population diagram}

Another approach to finding the relevant excitation mechanism is to assume thermal excitation. The ro-vibrational levels are then populated according to the Boltzmann equation. This can be illustrated by a population diagram showing the observed population density versus the energy of the upper level. If all values lie on a straight line, the gradient is proportional to the temperature of the molecular gas. The population density can be derived from the observed column density according to Lester et al. (1988):

$$
N_{\text {col }}=\frac{f}{A_{u l}} \frac{\lambda}{h c} \times \frac{4 \pi}{\Omega_{\text {aperture }}},
$$

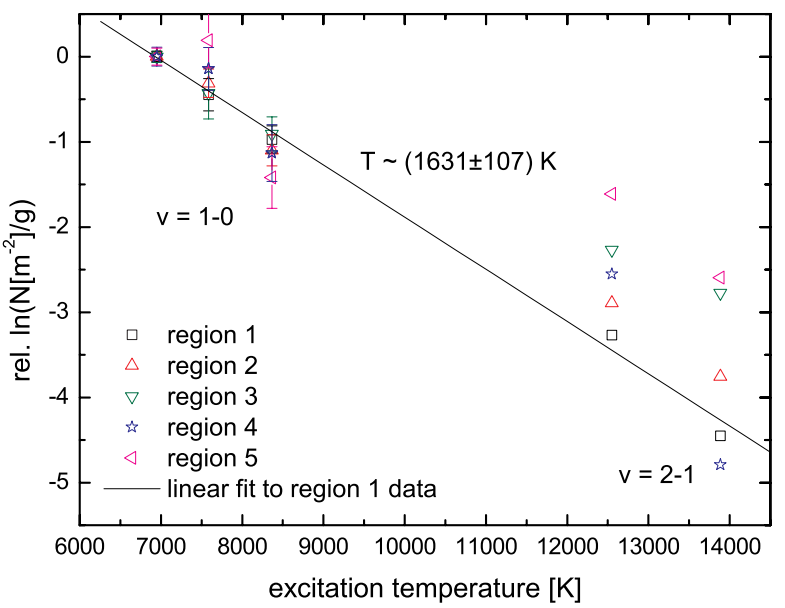

Fig. 10. $\mathrm{H}_{2}$ level population diagram relative to $1-0 \mathrm{~S}(1)$ for the the five regions of Fig. 2a. All $v=2-1$ fluxes are upper limits. A linear fit to the region 1 data is presented and yields an excitation temperature of about $T_{\text {ex }}=(1631 \pm 107) \mathrm{K}$ (cf. Fig. 9).

where $[f]=\mathrm{W} \mathrm{m}^{-2}$ is the observed line flux, $A_{u l}$ the transition probability (taken from Wolniewicz et al. 1998), $\lambda$ the rest frame line wavelength, $h$ the Planck constant, and $c$ the velocity of light. The additional factor $4 \pi / \Omega_{\text {aperture }}$ takes the spatial distribution into account. The Boltzmann distribution for thermal equilibrium can be written as

$\frac{N^{\prime}}{N^{\prime \prime}}=\frac{g_{J}^{\prime}}{g_{J}^{\prime \prime}} \mathrm{e}^{-\Delta E / k T}$,

where $N^{\prime}$ and $N^{\prime \prime}$ denote the column densities at the corresponding levels, $g_{J}^{\prime}$ and $g_{J}^{\prime \prime}$ are the statistical weights, and $T$ the temperature of the thermal equilibrium. If the $\mathrm{H}_{2}$ is non-thermally excited, only lines within one rotational level (e.g. $v=1-0$ ) fall on a straight line.

The population diagram Fig. 10 for the $\mathrm{H}_{2}$ lines of Mrk 609 shows that, within their errors, the different transitions fall on a straight line. From this we estimate a kinetic temperature for the nuclear region of about $1631 \pm 107 \mathrm{~K}$. It has to be noted that the $v=2-1$ fluxes are upper limits. For region 1 these values are consistent with the $v=1-0$ kinetic temperature. For regions $2-4$, the scatter with respect to a linear relationship is larger and the $v=2-1$ values tend to show lower excitation temperatures. Nevertheless, the temperatures found in the population diagram agree with the temperatures indicated by the solid curve in Fig. 9. A stronger non-thermal contribution might be possible for region 5 .

\subsection{Stellar absorption features}

The $H+K$ spectra of all five regions show stellar absorption features like the $\mathrm{CO}(6-3) 1.62 \mu \mathrm{m}$ and $\mathrm{CO}(2-0) 2.29 \mu \mathrm{m}$, as well as Si I $1.59 \mu \mathrm{m}, \mathrm{Mg}$ I $1.5,1.71 \mu \mathrm{m}$, and Na I $2.206 \mu \mathrm{m}$ (see Fig. 12). The details of the stellar content will be discussed in a subsequent paper, but one can get initial insight into the stellar populations by using the ratio of equivalent widths $(E W)$ of $\mathrm{CO}(6-3)$ and $\mathrm{Si} 1.59 \mu \mathrm{m}$ to indicate the temperature of latetype stars (Origlia et al. 1993; Ivanov et al. 2004). CO(6-3) grows rapidly from early $\mathrm{K}$ to late $\mathrm{M}$ stars, while SiI varies only very slowly with temperature. Table 4 lists the ratio for all regions. The measured ratios correspond to M-type giants (see Fig. 5b in Origlia et al. 1993). One has to take into account that 


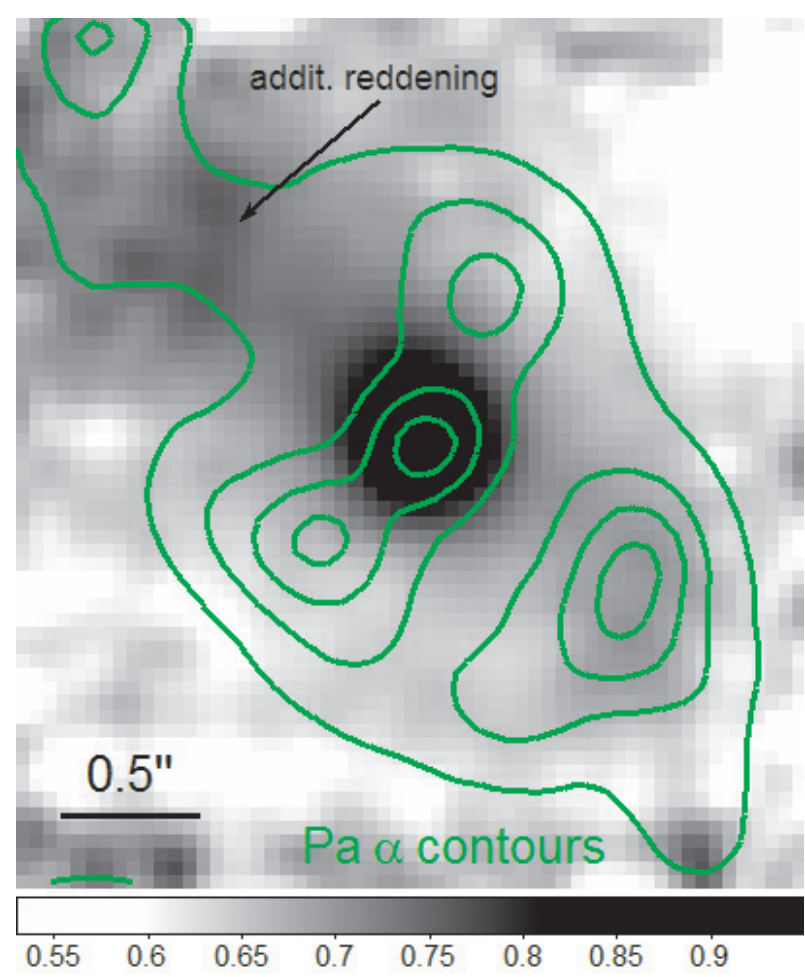

Fig. 11. Color map derived from continuum fluxes measured at the 2MASS effective $H$ and $K$ s band wavelengths and zero points. Darker greyscales indicate redder colors with $H-K$ ranging from 0.5 to 1 . Contours correspond to $\mathrm{Pa} \alpha$ emission displayed in Fig. 2a.

in active galaxies these stellar features can be substantially diluted by non-stellar nuclear emission. This effect can be recognized by comparing spectra of regions 1 and 2 of both panels in Fig. 12. While being very similar in the $H$ band, the nuclear $K$-band spectrum is significantly reddened. However, we do not go on to consider the effect at this point.

The $\mathrm{CO}(2-0)$ index can be used to estimate the age of the stellar population (Origlia \& Oliva 2000). As outlined in Ramos Almeida et al. (2006), we use the Ivanov CO index (Ivanov et al. 2004) and convert it to a photometric CO index to read the approximate age from Fig. 1 of Origlia \& Oliva (2000). The photometric CO indices range from 0.1 to 0.15 . For solar metallicity this corresponds to ages between 80 and $150 \mathrm{Myr}$ for the Geneva tracks with the younger ages measured in regions 2 and 5. Such deep CO absorption is typically found in starburst galaxies (e.g. Fischer et al. 2006, and references therein). Note that the blue edge of the $\mathrm{CO}(2-0)$ bandhead is influenced by atmospheric $\mathrm{OH}$ emission, and the change in index is on the order of $20 \%$. Thus, the stellar ages might become shorter by a few 10 Myr.

The NaI absorption is commonly found in late-type stars. Model calculations by Davies et al. (2005) show that the $E W$ of $\mathrm{Na}$ I typically ranges between 2 and $3 \AA$. Their models include AGB phases that have a significant influence on the depth of the $\mathrm{Na}$ I feature (see Fig. 7 of Davies et al. 2005). Indeed, the $E W \mathrm{~s}$ range between 2.5 (for region 1) and 3.8 (for region 5), which is consistent with the ages found from the $\mathrm{CO}(2-0)$ index. Both results agree with the young stars found by Rudy et al. (1988) from their UV/optical spectroscopy.
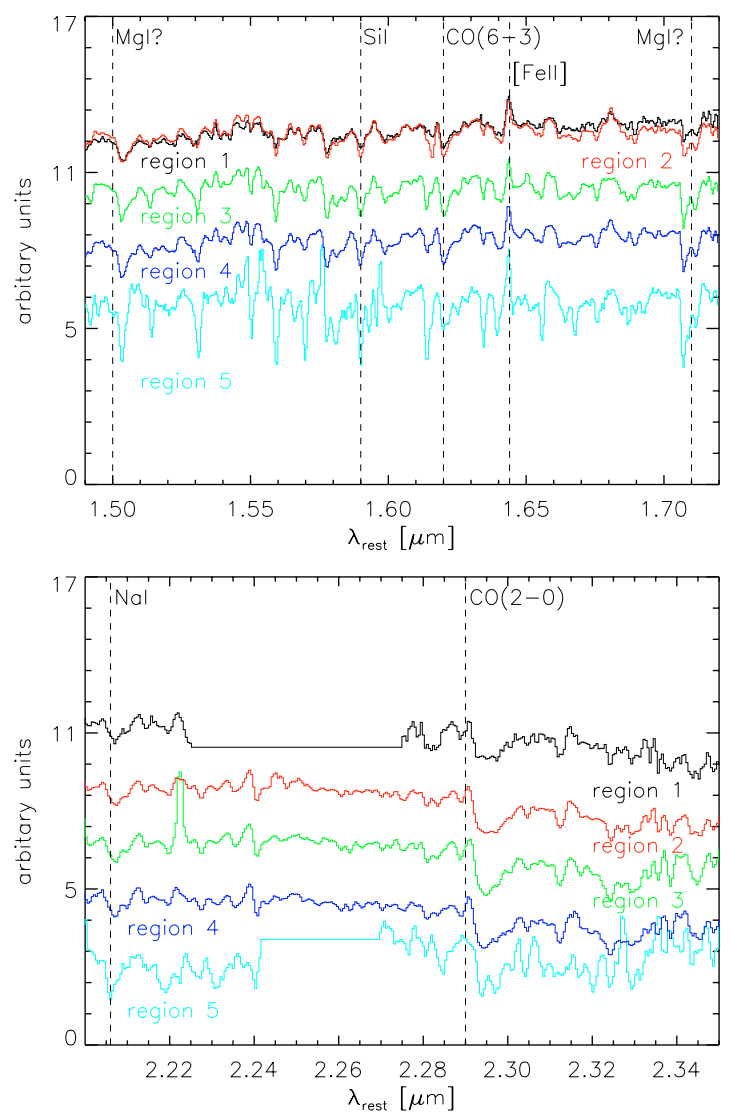

Fig. 12. Stellar absorption features in the $H$-band (upper panel) and $K$-band (lower panel) parts of the spectra extracted from the five regions of Fig. 2a. The fluxes of the $H$ and $K$ spectra are scaled to the flux at $2.1 \mu \mathrm{m}$ of region 1 . Then the spectra were shifted by an arbitrary amount for better visibility. The $K$-band part of region 1 between 2.225 and $2.275 \mu \mathrm{m}$ is not useable because of a detector defect and has been replaced by a flat line. The same situation applies for region 5 between 2.241 and $2.27 \mu \mathrm{m}$.

Table 4. Measurements of stellar absorption lines.

\begin{tabular}{cccc}
\hline \hline Region & $E W(\mathrm{CO}(6-3)) / E W(\mathrm{SiI})$ & $\mathrm{CO}(2-0)$ index & $\begin{array}{c}E W(\mathrm{NaI}) \\
{[\AA]}\end{array}$ \\
\hline 1 & 0.31 & 0.09 & 2.5 \\
2 & 0.28 & 0.19 & 2.8 \\
3 & 0.26 & 0.14 & 3.0 \\
4 & 0.31 & 0.13 & 3.0 \\
5 & 0.24 & 0.17 & 3.8 \\
\hline
\end{tabular}

\section{4. $\mathrm{CO}(1-0)$ observations}

Another ingredient in the starburst/Seyfert picture is the presence of fuel in the form of molecular gas. Giant molecular clouds are the birthplaces of stars. The dense gas is dissipative and galaxy interaction is believed to channel huge amounts of molecular gas towards the nucleus of the interacting galaxies, providing fuel for the nuclear activity (e.g. Springel et al. 2005). Infrared luminous AGN $\left(L_{\mathrm{IR}}>10^{10} \mathrm{erg} \mathrm{s}^{-1}\right)$ are found to be rich in molecular gas (e.g. Evans et al. 2001, 2005, and references therein) with gas masses of up to $M_{\mathrm{H}_{2}} \sim 10^{10} M_{\odot}$. Maiolino et al. (1997) explain the more intense star formation found in Seyfert 2 galaxies with a significantly higher fraction of asymmetric morphologies than in Seyfert 1 and field galaxies. As discussed in Sect. 1.1, there is compelling observational and theoretical evidence that bars efficiently redistribute angular momentum in 


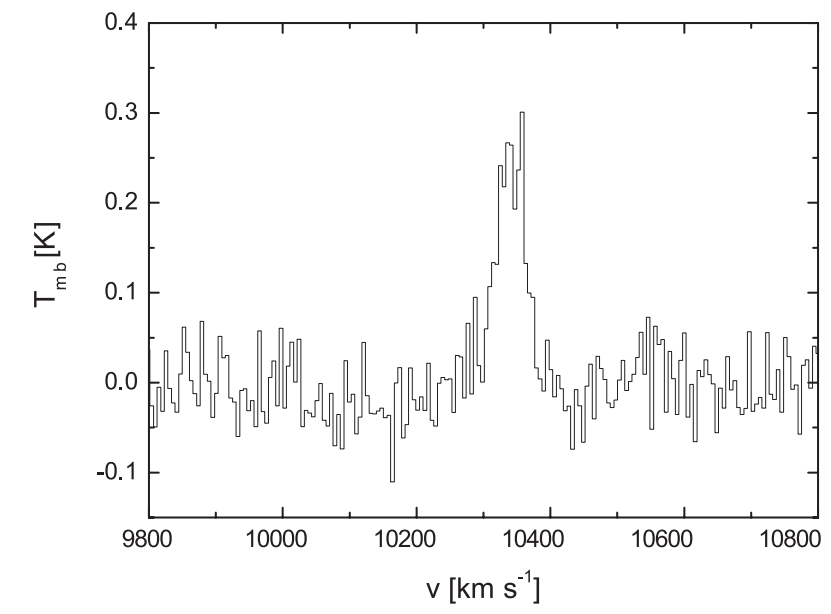

Fig. 13. Observed-frame $\mathrm{CO}(1-0)$ spectrum of Mrk 609.

galaxies and cause gas to flow inwards into the circumnuclear region (inner $1-2 \mathrm{kpc}$ ), giving rise to starburst and nuclear activity (Kormendy \& Kennicutt 2004; Jogee et al. 2005). A major result from the Nuclei of Galaxies (NUGA) project carried out at the Plateau de Bure interferometer is the wide variety of circumnuclear disk morphologies on the $100 \mathrm{pc}$ scale associated with the AGN (García-Burillo et al. 2005; and also Schinnerer et al. 2000, for an integrated CO/NIR study of NGC 1068). It is believed that on these scales, secondary perturbations modes appear to take over and are responsible for channeling gas towards the center of the galaxy. Still the scales are large compared with nuclear scales. The actual fueling of the central engine is believed to be supported by viscous flow of the material delivered by the secondary perturbations (Duschl \& Strittmatter 2004).

During an observing campaign of cluster galaxies with the Nobeyama $45 \mathrm{~m}$ telescope in March 2005, we also observed Mrk 609 in ${ }^{12} \mathrm{CO}(1-0)$. The baseline-subtracted, main-beamefficiency corrected $\mathrm{CO}(1-0)$ spectrum is presented in Fig. 13. The beam with a size of about $15^{\prime \prime}$ essentially covers the entire visual part of the galaxy (cf. Fig. 1). The FWHM of the CO line is $51 \pm 4 \mathrm{~km} \mathrm{~s}^{-1}$ and the line-integrated flux, corrected for main-beam efficiency, is $I_{\mathrm{CO}}=(14 \pm 1) \mathrm{K} \mathrm{km} \mathrm{s}^{-1}$. The narrow line width is reminiscent of the small inclination of Mrk 609. According to Eq. (2) in Solomon et al. (1992), the line luminosity results in $L_{\mathrm{CO}}^{\prime}=(1.5 \pm 0.1) \times 10^{9} \mathrm{~K} \mathrm{~km} \mathrm{~s}^{-1} \mathrm{pc}^{2}$. We can now estimate the $\mathrm{H}_{2}$ mass, assuming optically thick and thermalized emission, originating from gravitationally bound molecular clouds (Evans et al. 2005):

$\alpha=\frac{M\left(\mathrm{H}_{2}\right)}{L_{\mathrm{CO}}^{\prime}} M_{\odot}\left(\mathrm{K} \mathrm{km} \mathrm{s}^{-1} \mathrm{pc}^{2}\right)^{-1}$.

The conversion factor $\alpha$ lies between 2 and 5 (Radford et al. 1991). Here we adopt $\alpha=4$, resulting in $M\left(\mathrm{H}_{2}\right)=(6.0 \pm 0.4) \times$ $10^{9} M_{\odot}$, which is on the high end of the range of masses found in AGN (e.g. Rigopoulou et al. 1997).

The gas mass can be compared with the dust mass derived from the IRAS $60 \mu \mathrm{m}$ and $100 \mu \mathrm{m}$ fluxes $\left(f_{60}=2550 \mathrm{mJy}\right.$, $f_{100}=4760$ mJy; Rudy \& Rodriguez-Espinosa 1985). The infrared emission originates from warm dust, heated either by star-formation or by the active nucleus. Following Evans et al. (2005), the dust temperature results in $T_{\text {dust }} \approx 47 \mathrm{~K}$, and the corresponding dust mass in $M_{\text {dust }} \approx 1.1 \times 10^{7} M_{\odot}$. The gasto-dust ratio, $\sim 545$, is comparable to IRAS-detected spiral and luminous infrared galaxies (Young \& Scoville 1991). Next, we calculate the infrared luminosity using the formula given in
Solomon et al. (1997). Tutui et al. (2000) furthermore show that an IRAS $K$-correction for this type of galaxy is not necessary. The color correction, $r$, lies between 1.5 and 2.1 (Solomon et al. 1997). Assuming $r=1.8$, the infrared luminosity calculates as $L_{\mathrm{FIR}} \approx 10^{11} L_{\odot}$, which is typical for an infrared luminous galaxy.

The FIR luminosity of (inactive) galaxies is interpreted as a measure of the number of visible and UV photons, thus measuring the number of high-mass stars. This allows us to estimate the global star-formation rate (SFR) from the IRAS fluxes using Eqs. (7) and (8) in Hopkins et al. (2003). The global SFR of Mrk 609 amounts to $\sim 30 M_{\odot} \mathrm{yr}^{-1}$, on the other hand, $\mathrm{CO}(1-0)$ measures the cold molecular gas supplying the star formation. The ratio between FIR and CO luminosity, therefore, is a measure of the star formation efficiency (SFE; cf. Eckart et al. 1994). The global SFE is on the order $L_{\mathrm{FIR}} / M_{\mathrm{H}_{2}} \approx 17 L_{\odot} M_{\odot}^{-1}$, which is again on the high end of the SFEs of non-interacting galaxies. Such SFRs and SFEs are also often found in interacting systems (Solomon \& Sage 1988). Stars thus appear to be formed very efficiently in Mrk 609 and the gas depletion time is short $\left(\sim 3 \times 10^{8} \mathrm{yr}\right)$.

We can also estimate an SFR using the $21 \mathrm{~cm}$ radio flux. For Mrk 609, the NRAO VLA Sky Survey (NVSS) $21 \mathrm{~cm}$ flux $f_{21 \mathrm{~cm}}=30$ mJy yields a luminosity of $L_{21 \mathrm{~cm}}=8.3 \times 10^{22} \mathrm{~W} \mathrm{~Hz}^{-1}$. According to Eqs. (1) and (2) of Hopkins et al. (2003), the radio derived SFR amounts to $\sim 46 M_{\odot} \mathrm{yr}^{-1}$. This is somewhat higher than the FIR-derived SFR. Hopkins et al. (2003) find a tight correlation between radio and FIR flux. The differences in the derived SFRs could be caused by a stronger AGN contribution (e.g. jet) at the $21 \mathrm{~cm}$ wavelengths.

\subsection{The starburst/AGN connection in Mrk 609}

In contrast to previous observations of Mrk 609, we have now spatially resolved the inner circumnuclear environment on the $270 \mathrm{pc}$ scale. This allows us to study the relative contributions and importance of nuclear and off-nuclear (H II regions, late-type stars) emission.

Analogously to the optical (e.g. Baldwin et al. 1981; Veilleux \& Osterbrock 1987), Rodríguez-Ardila et al. (2004, 2005) emphasize the use of the line ratios $[\mathrm{Fe}$ II $] / \mathrm{Pa} \beta$ and $1-0 \mathrm{~S}(1) / \mathrm{Br} \gamma$ to distinguish between starbursts, AGN, and LINERs (low ionization nuclear emission-line region galaxy). They find that objects with either one of the ratios being lower than 2 were predominantly identified as Seyferts. Starburst galaxies, on the other hand, are located in a region where both ratios are lower then 0.4 , while LINERs have both ratios higher than 2 (Fig. 14).

In this context, the ratios describe the transition from pure shock excitation driven by supernova remnants (upper right corner of the diagram) to purely ionizing radiation powered by star formation (lower left corner). For illustration purposes we plot the data from Rodríguez-Ardila et al. (2005) as open symbols in Fig. 14. Starbursts from Dale et al. (2004) populate the lower left region of the diagram. Seyfert 1 and 2 galaxies from Rodríguez-Ardila et al. (2005) populate the middle part, whereas LINERs (Larkin et al. 1998) can be found in the upper right corner. The emission of the supernova IC 443, on the other hand, is believed to be driven by shocks (Graham et al. 1987). The location of AGN (powered by X-ray heating) between these two extremes has not been fully understood yet. But most probably it is the result of a power-law continuum illuminating a slab of gas (see below and Rodríguez-Ardila et al. 2005).

It can be seen from Figs. $2 \mathrm{~b}$ and $4 \mathrm{c}$ that the [Fe II] emission is extended towards regions $2-4$. This extent is about perpendicular to the $\mathrm{H}_{2}$ one. Moreover, the radio contours are 


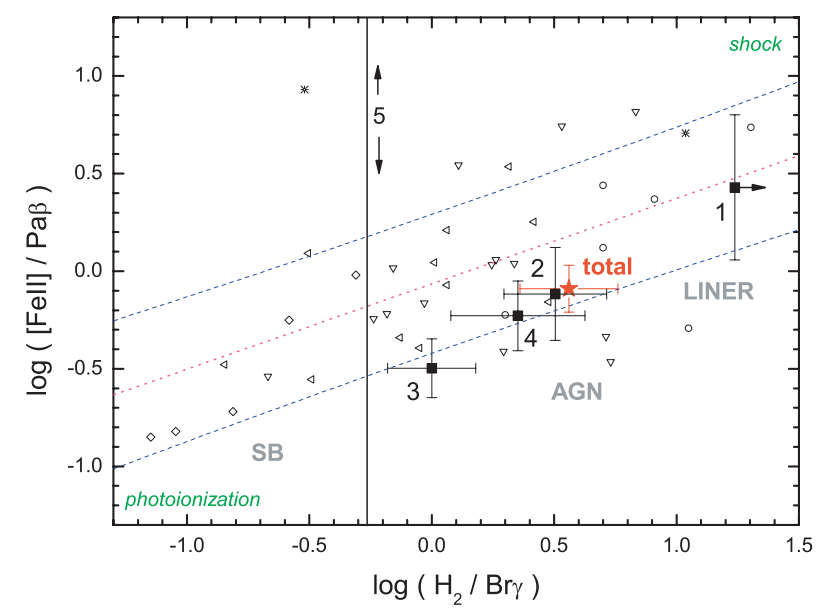

Fig. 14. Line ratios of [Fe II] $1.257 \mu \mathrm{m} / \mathrm{Pa} \beta$ and $1-0 \mathrm{~S}(1) 2.121 \mu \mathrm{m} / \mathrm{Br} \gamma$. Activity types (starburst, AGN, LINER) are indicated. Filled symbols represent our measurements of the five regions of Mrk 609, as well as for the total FOV (red star). Because of missing $J$-band data, region 5 is only accurately located along $\mathrm{H}_{2} / \mathrm{Br} \gamma$. Open symbols correspond to literature values: rhombs represent starburst galaxies from Dale et al. (2004), left triangles Seyfert 1 galaxies from Rodríguez-Ardila et al. (2004), headlong triangles Seyfert $2 \mathrm{~s}$ from Rodríguez-Ardila et al. (2005), circles LINERs from Larkin et al. (1998), and asterixs supernovae also from Larkin et al. (1998). The diagram is interpreted as displaying the transition from pure photoionization (lower left corner) to pure shock driven (upper right) emission (Rodríguez-Ardila et al. 2005). Assuming a linear relationship between the two line ratios, the dotted magenta line represents a linear fit to the literature data points. The blue dashed lines represent the $1 \sigma$ prediction band of the fit.

approximately congruent with the [Fe II] distribution. The NIR line-ratios measured in the five regions fall into different parts of the NIR diagnostics diagram (Fig. 14). The calibration uncertainties discussed above play no role at this point, since the line ratios are calculated within a spectral band. Our data points clearly follow a trend from a Liner-like value at the nucleus to a starburst-like value at the most distant region 5 . The circumnuclear regions 2-4 fall in the domain of potentially mixed excitation. We discuss the situation for the individual regions below. Under the assumption that the literature values follow a linear relationship between the line ratios (from photoionizationto shock-driven excitation, see Larkin et al. 1998), we carried out a linear regression shown in Fig. 14 (correlation coefficient $R \sim 0.6$ and a probability of $R=0$ of about $10^{-4}$ ) with its $1 \sigma$ prediction band. This fit, however, is only intended to guide the eye. A firm correlation has not been established yet. Notice that our data fall somewhat below the linear relation, but still within the prediction band. Incorrect estimations of the continuum levels during the measurement of emission line fluxes might in part be responsible for this trend. On the other hand, M-type giants are the dominating population in the NIR (Sect. 3.3), and stellar absorption can therefore influence the flux measurement of emission lines (e.g. Harrison et al. 1998, who found that stellar absorption plays an important role in their observation). The study of Larkin et al. (1998) used larger apertures compared to our work from which they extracted spectra. In this case a considerable part of the host galaxy emission can modify the emissionline measurements. On the other hand, Rodríguez-Ardila et al. $(2005,2004)$ use nuclear apertures of sizes comparable to our work. The latter study, however, does not consider circumnuclear stellar absorption features at the position of the emission lines, which can influence the results.

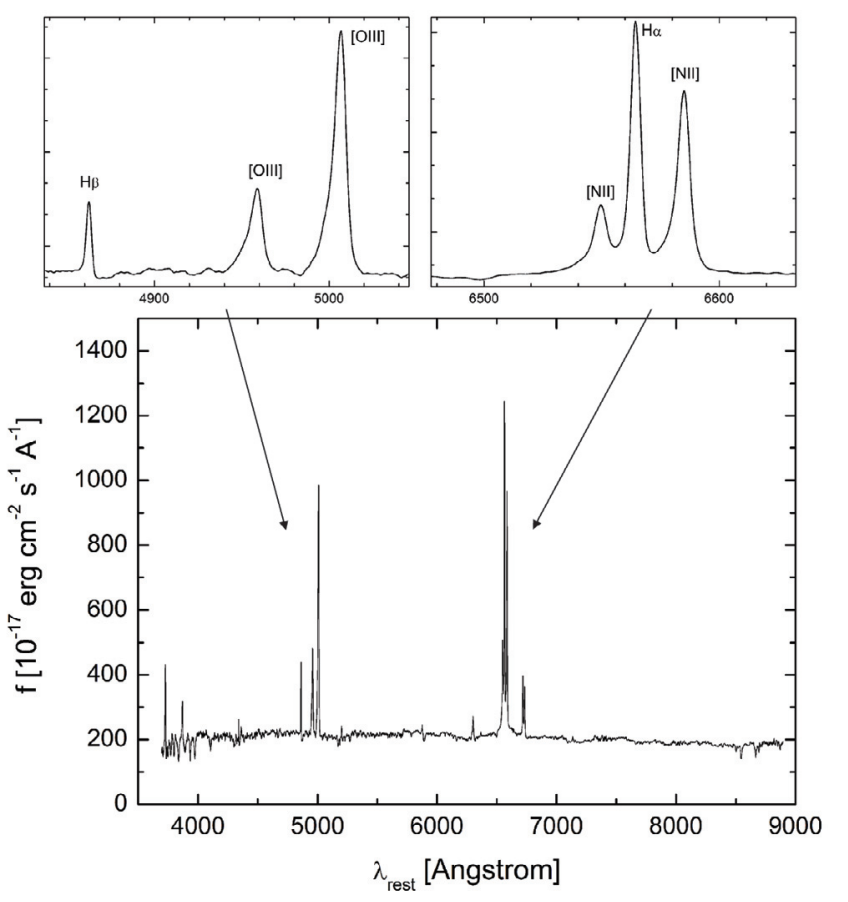

Fig. 15. Rest frame SDSS spectrum (from SDSS DR4 Adelman-McCarthy 2005). The upper insets are close-ups of the $\mathrm{H} \beta$ and $\mathrm{H} \alpha$ lines. The spectra are measured through a $3^{\prime \prime}$ diameter fiber.

Visual inspection of the spectra of late-type stars (Wallace et al. 2000; Ivanov et al. 2004 in $J$ and $H, K$, respectively) shows that there is no significant stellar absorption at the position of the emission lines. Atmospheric residuals might account for an uncertainty in flux on the order of $20 \%$, as discussed in Sect. 3.3.

The nuclear region interestingly falls into the LINER regime. This contrasts with the optical classification as Sy1.8 by Osterbrock (1981) or from analysis of the SDSS spectrum (Fig. 15). This discrepancy may be due to aperture effects, since the SDSS spectrum has been measured in a $3^{\prime \prime}$ diameter fiber covering the total FOV of our NIR observations. The total narrow-line flux of the H-recombination lines is dominated by the extra-nuclear regions. The LINER character might therefore be hidden in the large-aperture optical spectrum. Such effects are also discussed by Kauffmann et al. (2003). This can be tested in the present case by computing the flux ratio of the line fluxes integrated over the SINFONI FOV $(\sim 1.3 \mathrm{kpc} \times 1.3 \mathrm{kpc})$. Indeed, the position in the diagnostics diagram is shifted towards values typically found for Seyfert galaxies (Fig. 14). This demonstrates the importance of high angular resolution in studying the nature of activity in galaxies. It is furthermore interesting that the offnuclear emission regions populate a region in the diagnostic diagram where AGN are usually found. This indicates that in these regions both shock and photoionization are important.

Regions 2-4 have line ratios typical of AGN, although the $[\mathrm{Fe} \mathrm{II}] / \mathrm{Pa} \beta$ ratio is close to starburst values. Region 5 has a $1-0 \mathrm{~S}(1) / \mathrm{Br} \gamma$ ratio among those of starbursts, although the $[\mathrm{Fe} \mathrm{II}] / \mathrm{Pa} \beta$ ratio is unconstrained because of the lack of $J$ band spectral information. The overlay of the $\mathrm{Pa} \alpha$ with the $6 \mathrm{~cm}$ radio map (Fig. 3d) from Ulvestad \& Wilson (1984) shows radio emission in all five regions. The two-point spectral index $\alpha_{6 \mathrm{~cm} / 21 \mathrm{~cm}} \approx 1.3$, assuming a power-law of the form $F_{v} \propto v^{-\alpha}$, is on the high end of values observed for synchrotron radiation (Ulvestad \& Wilson 1984; Filho et al. 2000). Such a steep spectral index is commonly found in star-forming late-type galaxies 
Table 5. Emission line fluxes from SDSS spectrum (Fig. 15).

\begin{tabular}{ccc}
\hline \hline Line & $\begin{array}{c}\text { flux } \\
{\left[10^{-17} \mathrm{Wm}^{-2}\right]}\end{array}$ & $\begin{array}{c}F W H M \\
{\left[\mathrm{~km} \mathrm{~s}^{-1}\right]}\end{array}$ \\
\hline [O II] 3727 $\AA$ & $1.9 \pm 0.1$ & 480 \\
$\mathrm{H} \beta$ narrow & $0.80 \pm 0.02$ & 190 \\
[O III] $5007 \AA$ & $7.7 \pm 0.1$ & 400 \\
[O I] 6300 $\AA$ & $0.71 \pm 0.04$ & 365 \\
$\mathrm{H} \alpha$ narrow & $4.9 \pm 0.3$ & 220 \\
$\mathrm{H} \alpha$ broad & $8.1 \pm 0.4$ & 3170 \\
{$[\mathrm{~N}$ II] 6585 $\AA$} & $4.5 \pm 0.3$ & 300 \\
\hline
\end{tabular}

(Condon 1992). Together with the 1-0S(1)/Br $\gamma$ value, this indicates the starburst nature of the emission region 5 . As mentioned above, the three other off-nuclear ratios populate the transition region from starburst to AGN in the diagram. At a projected distance of about $420 \mathrm{pc}$, regions 2 and 4 still show the strong influence of the (LINER) active nucleus. The jet-like extension of the $6 \mathrm{~cm}$ emission towards regions 2 and 4 might also suggest the importance of outflows and associated shocks that are able to excite [Fe II] and/or $\mathrm{H}_{2}$. Region 3 at a projected distance of about $630 \mathrm{pc}$ lies even closer to the starburtst regime. This is expected, since the nuclear influence should decrease at larger separations.

In their study of LINERs, Larkin et al. (1998) investigated several multi-wavelength correlations. They found that LINERs and Seyferts do not follow the correlations between the IRAS $25 \mu \mathrm{m}$ to $60 \mu \mathrm{m}$ index and $\mathrm{H}_{2} / \mathrm{Br} \gamma$ and $[\mathrm{OI}] / \mathrm{H} \alpha$ found by Mouri \& Taniguchi (1992) for starburst galaxies. The IRAS $25 \mu \mathrm{m}$ and $60 \mu \mathrm{m}$ fluxes are sensitive to warm $(30-50 \mathrm{~K})$ and hot $(100-150 \mathrm{~K})$ dust. Seyfert galaxies typically show a shallow index. Therefore this index can be used as a Seyfert identifier (de Grijp et al. 1992). LINERs show a wide spread in the IRAS index, whereas they only show little variation in the $\mathrm{H}_{2} / \mathrm{Br} \gamma$ and $[\mathrm{O} \mathrm{I}] / \mathrm{H} \alpha$ ratios (Larkin et al. 1998). The values for Mrk $609(\alpha(25: 60)=-1.9 ;[\mathrm{OI}] / \mathrm{H} \alpha \approx 0.15$; $\mathrm{H}_{2} / \mathrm{Br} \gamma \approx 0.55$ for the total FOV or $\mathrm{H}_{2} / \mathrm{Br} \gamma \approx 1.23$ for the nuclear region) fall between the starburst correlation and the region occupied by LINERs. Note that the optical line-ratio is derived from the $3^{\prime \prime}$ diameter aperture SDSS spectrum, whereas the NIR line-ratio is calculated for region 1 and for the total FOV. Nevertheless, a trend towards LINER values can be recognized at both wavelengths. Besides the aperture effects mentioned above, considerable variability in the emission has been detected (Rudy et al. 1988; Pappa et al. 2002), which can be combined with the duty cycle hypothesis of Eracleous et al. (1995). The authors propose recurring accretion events that enhance the non-stellar continuum and generate Seyfert characteristics like broad emission lines. After the accretion event, the ionizing flux drops and the high-ionization states weaken, whereas the lower-ionization lines persist much longer, because of longer decay and light crossing times compared to the broadline region. In this respect, Mrk 609 might approach a quiescent activity state in which the relative contribution of lowerionization and shock-driven emission is enhanced with respect to photoionization. The starburst, since farther away from the nucleus, remains constant. Unfortunately, no multi-epoch/multiwavelength observations of Mrk 609 exist that could substantiate this scenario; and due to the calibration problems we cannot compare our line fluxes with the NIR measurements of Goodrich (1990) and Rix et al. (1990).

The panchromatic properties of Mrk 609 reveal its composite nature. In Fig. 16 we plot the spectral energy distribution (SED)

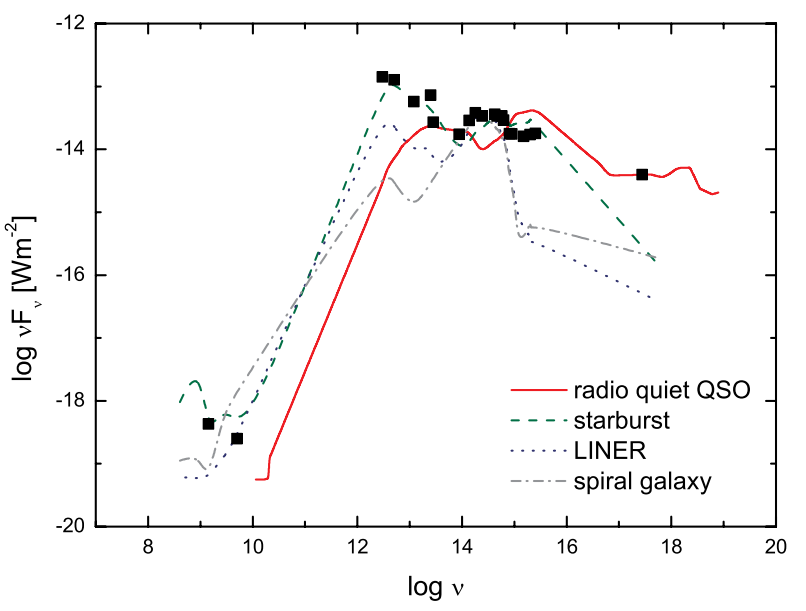

Fig. 16. Spectral energy distribution of Mrk 609 constructed from literature values (Rudy et al. 1988; Polletta et al. 1996). The values are compared with average SEDs from normal galaxies, starbursts, LINERs (Schmitt et al. 1997), and radio-quiet QSOs (Elvis et al. 1994).

and compare it to average SEDs of starburst, LINER, and spiral galaxies (taken from Schmitt et al. 1997). Also shown is the average SED of radio-quiet quasars from Elvis et al. (1994). It can be seen that the X-ray flux is nicely represented by an AGNlike contribution. Pappa et al. (2002) estimate from the FIR luminosity that a starburst can only contribute up to $50 \%$ of the X-ray luminosity. But the AGN itself cannot explain the longer wavelength fluxes, which appear to be a combination of the three other contributions. Only starbursts can produce the strong FIR fluxes. The LINER SED also fits nicely into this picture. Note that Mrk 609 is missing a significant big blue bump, which is also absent in low-luminosity AGN and might be explained by low accretion-rate models (Ho 1999). Furthermore, Ward et al. (1988) found a strong correlation between the broad $\mathrm{H} \alpha$ and the $2-10 \mathrm{keV}$ X-ray luminosity as a measure for the ionizing strength of the active nucleus. Pappa et al. (2002) find, that the $\mathrm{H} \alpha$ emission from the large aperture spectrum is underluminous by a factor of about 40 and suggest a deficit of ionizing photons. This is agrees with the missing UV-bump in the SED. Another possibility is the variability of the nonstellar continuum over the $15 \mathrm{yr}$ between optical and X-ray emission.

The nuclear continuum and emission-line morphology is not symmetric and possibly resembles that of a weak nuclear bar (Figs. 2d, 4a). The emission peaks of the $\mathrm{Pa} \alpha$ map are aligned with the major and minor axes of the bar. In addition, the slight elongation of the $6 \mathrm{~cm}$ radio emission towards regions 2 and 4 (Fig. 3d) could be interpreted as a jet, which might in part be responsible for the heating of regions 2 and 4 (e.g. van Breugel \& Dey 1993). The interpretation is still not clear from our data. On the other hand, the clumpy structure of the ISM seen in the recombination line maps might also resemble a ring-like structure or parts of a nuclear spiral. Such structures have been uncovered in both the observation (e.g. Erwin \& Sparke 2002; Martini et al. 2003; Emsellem et al. 2003) and simulation (e.g. Englmaier \& Shlosman 2000; Maciejewski 2004) of several quiescent and active galaxies. Torques associated with the gas response to the stellar-bar potential allow considerable amounts of gas to flow closer to nuclear regions $\left(\sim 1 M_{\odot} \mathrm{yr}^{-1}\right.$ on time scales of $\sim 10^{8} \mathrm{yr}$; Englmaier \& Shlosman 2004) where viscous processes take over the dissipation of angular momentum (Duschl $\&$ Strittmatter 2004). From the symmetrical appearance with respect to the bar's minor axis, we might expect a northeastern 
counterpart for region 3, which is missing, however, in the hydrogen emission-line maps. Nevertheless, the reddening map exhibits some additional reddening at the position of the proposed counterpart. The velocity maps of the hydrogen recombination line do not show any dynamical behavior, which is expected due to the face on view of the galaxy and the limited spectral resolution. Therefore, we are currently not in a position to favor any of the above models.

\section{Summary and conclusions}

Mrk 609 was chosen from a sample of AO-suitable optical counterparts of luminous ROSAT X-ray AGN at low to intermediate redshifts (Zuther et al. 2004, 2005). It is one of the lowest redshift objects in the sample and is therefore best-suited for initial integral field observations.

We have presented first results on the circumnuclear structure of the starburst/Seyfert composite Mrk 609 in the NIR using imaging spectroscopy with SINFONI. The morphology is complex, and the continuum images reveal a bar-like structure. The distribution of hydrogen recombination emission $(\mathrm{Pa} \alpha, \mathrm{Pa} \beta$, $\mathrm{Br} \gamma)$ is clumpy and peaks at the tip where the potential bar meets the spiral arms and in regions along the minor axis. Bars can account for the angular momentum transfer necessary for fueling nuclear activity. The $6 \mathrm{~cm}$ VLA emission is also extended in the latter direction. Whether this emission is associated with a jet or with resonances in the bar potential, is unclear. The presence of nuclear broad $\mathrm{Pa} \alpha$ and $[\mathrm{Si} \mathrm{VI}]$ are clear indicators of the accretion of matter onto a nuclear super-massive black hole.

The analysis of molecular hydrogen and [Fe II] emission indicates the importance of shock heating, although X-ray heating by the nucleus and non-thermal contributions is possible. The distribution of molecular hydrogen follows the continuum shape, while that of [Fe II] is aligned with the minor axis of the continuum and with the radio contours, as well as with the H-recombination line peaks. The nucleus itself shows signs of LINER activity, which can be recognized by its high [Fe II] $/ \mathrm{Pa} \beta$ and $\mathrm{H}_{2} / \mathrm{Br} \gamma$ values. Our integral-field data clearly resolve the nuclear and starburst activity in the central kilo parsec. Extinction appears to play no crucial role in this region, since the $\mathrm{H}$-recombination line ratios are consistent with unreddened case-B values. This is supported further by the strong Ly $\alpha$ emission. However, already small amounts of extinction have considerable effects at visible wavelengths, as can be seen in the flocculent morphology of Fig. 1. Continuum reddening in the NIR, on the other hand, can already be caused by small amounts of warm/hot dust. The reddening of the continuum found toward the nucleus and the other regions is typical of AGN and starforming regions where the dust reprocesses UV radiation from hot stars or the active nucleus.

We found large amounts of cold molecular gas, which provides the fuel for the star-forming activity. M-type giants are the dominating stellar population in the NIR, which indicates starburst ages of about $100 \mathrm{Myr}$ (for a single burst). The nuclear stellar absorption features are considerably diluted by the nonstellar continuum.

Our LINER classification, together with the published variability of the non-stellar NIR emission, might be explained by the duty-cycle hypothesis in which short-lived accretion events occur periodically and lead to the appearance of Seyfert features in the high state and of low-ionization (shock driven) emission features in the low state.

The X-ray emission can account for some of the $\mathrm{H}_{2}$ excitation. This might also explain the higher $\mathrm{H}_{2} / \mathrm{Br} \gamma$ ratios for the off-nuclear regions than expected for starbursts. The [Fe II] emission, however, does not seem to be excited by X-rays. Shock excitation along the southeast/northwest axis might be caused partly by a radio jet impinging into the ISM. The main driver for both species therefore appears to be shock excitation.

Our results show that high spatial resolution is vital for dissecting the nuclear and starburst activity in AGN. Morphological peculiarities, such as nuclear bars and nuclear starburst rings, are best detected at NIR wavelength, since the mass dominating stellar populations have their emission maximum in the NIR, and dust extinction is smaller than at visible wavelengths. In conjunction with high spectral resolution, it is possible to trace the stellar and gaseous nuclear kinematics in order to find important constraints for dynamical models describing the infall of matter into the nuclear regions (e.g. Fathi et al. 2005). This is a prerequisite for the study of composite systems in which nuclear star formation and Seyfert activity occur at similar levels of intensity.

Acknowledgements. The authors kindly thank the anonymous referee for fruitful comments and suggestions. This work was supported in part by the Deutsche Forschungsgemeinschaft (DFG) via grant SFB 494.

\section{References}

Adelman-McCarthy, J. K. 2005 [arXiv: astro-ph/0507711]

Almaini, O., Shanks, T., Boyle, B. J., et al. 1996, MNRAS, 282, 295

Alonso-Herrero, A., Ward, M. J., \& Kotilainen, J. K. 1996, MNRAS, 278, 902

Alonso-Herrero, A., Rieke, M. J., Rieke, G. H., \& Ruiz, M. 1997, ApJ, 482, 747 Baldwin, J. A., Phillips, M. M., \& Terlevich, R. 1981, PASP, 93, 5

Black, J. H., \& van Dishoeck, E. F. 1987, ApJ, 322, 412

Boyle, B. J., McMahon, R. G., Wilkes, B. J., \& Elvis, M. 1995, MNRAS, 276, 315

Brand, P. W. J. L., Toner, M. P., Geballe, T. R., et al. 1989, MNRAS, 236, 929

Calzetti, D., Kinney, A. L., \& Storchi-Bergmann, T. 1996, ApJ, 458, 132

Combes, F. 1996, in Unsolved Problems of the Milky Way, ed. L. Blitz, \& P. J. Teuben, IAU Symp., 169, 133

Condon, J. J. 1992, ARA\&A, 30, 575

Crenshaw, D. M., Kraemer, S. B., \& Gabel, J. R. 2003, AJ, 126, 1690

Dale, D. A., Roussel, H., Contursi, A., et al. 2004, ApJ, 601, 813

Davies, R. I., Sternberg, A., Lehnert, M. D., \& Tacconi-Garman, L. E. 2005, ApJ, 633, 105

de Grijp, M. H. K., Keel, W. C., Miley, G. K., Goudfrooij, P., \& Lub, J. 1992, A\&AS, 96, 389

Deo, R. P., Crenshaw, D. M., \& Kraemer, S. B. 2006, AJ, 132, 321

Draine, B. T. 1989, Interstellar extinction in the infrared (Infrared Spectroscopy in Astronomy, Proceedings of the 22nd Eslab Symposium held in Salamanca, Spain, 7-9 December, 1988, ed. B. H. Kaldeich, ESA SP-290, 93

Draine, B. T., \& McKee, C. F. 1993, ARA\&A, 31, 373

Draine, B. T., \& Woods, D. T. 1990, ApJ, 363, 464

Duschl, W. J., \& Strittmatter, P. A. 2004, in Studies of Galaxies in the Young Universe with New Generation Telescope, ed. N. Arimoto, \& W. J. Duschl, 205

Eckart, A., van der Werf, P. P., Hofmann, R., \& Harris, A. I. 1994, ApJ, 424, 627 Eisenhauer, F., Abuter, R., Bickert, K., et al. 2003, in Instrument Design and Performance for Optical/Infrared Ground-based Telescopes, ed. M. Iye, \& A. F. M. Moorwood, Proc. SPIE, 4841, 1548

Elvis, M., Wilkes, B. J., McDowell, J. C., et al. 1994, ApJS, 95, 1

Emsellem, E., Goudfrooij, P., \& Ferruit, P. 2003, MNRAS, 345, 1297

Englmaier, P., \& Shlosman, I. 2000, ApJ, 528, 677

Englmaier, P., \& Shlosman, I. 2004, ApJ, 617, L115

Eracleous, M., Livio, M., \& Binette, L. 1995, ApJ, 445, L1

Erwin, P., \& Sparke, L. S. 2002, AJ, 124, 65

Evans, A. S., Frayer, D. T., Surace, J. A., \& Sanders, D. B. 2001, AJ, 121, 3285

Evans, A. S., Mazzarella, J. M., Surace, J. A., et al. 2005, ApJS, 159, 197

Fathi, K., van de Ven, G., Peletier, R. F., et al. 2005, MNRAS, 364, 773

Filho, M. E., Barthel, P. D., \& Ho, L. C. 2000, ApJS, 129, 93

Fischer, S., Iserlohe, C., Zuther, J., et al. 2006, A\&A, 452, 827

Fischera, J., Dopita, M. A., \& Sutherland, R. S. 2003, ApJ, 599, L21

García-Burillo, S., Combes, F., Schinnerer, E., Boone, F., \& Hunt, L. K. 2005, A\&A, 441, 1011 
Gebhardt, K., Bender, R., Bower, G., et al. 2000, ApJ, 539, L13

Glass, I. S. 1999, Highlights of Astronomy

Glass, I. S., \& Moorwood, A. F. M. 1985, MNRAS, 214, 429

Goodrich, R. W. 1990, ApJ, 355, 88

Graham, J. R., Wright, G. S., \& Longmore, A. J. 1987, ApJ, 313, 847

Harrison, A., Puxley, P., Russell, A., \& Brand, P. 1998, MNRAS, 297, 624

Heckman, T. M., Kauffmann, G., Brinchmann, J., et al. 2004, ApJ, 613, 109

Hill, T. L., Heisler, C. A., Sutherland, R., \& Hunstead, R. W. 1999, AJ, 117, 111

Ho, L. C. 1999, ApJ, 516, 672

Ho, L. C., Filippenko, A. V., \& Sargent, W. L. W. 1997, ApJ, 487, 568

Hopkins, A. M., Miller, C. J., Nichol, R. C., et al. 2003, ApJ, 599, 971

Hunt, L. K., \& Malkan, M. A. 1999, ApJ, 516, 660

Hyland, A. R., \& Allen, D. A. 1982, MNRAS, 199, 943

Ivanov, V. D., Rieke, M. J., Engelbracht, C. W., et al. 2004, ApJS, 151, 387

Jogee, S., Scoville, N., \& Kenney, J. D. P. 2005, ApJ, 630, 837

Kauffmann, G., Heckman, T. M., Tremonti, C., et al. 2003, MNRAS, 346, 1055

Knapen, J. H. 2005, Ap\&SS, 295, 85

Kormendy, J., \& Kennicutt, R. C. 2004, ARA\&A, 42, 603

Kormendy, J., \& Richstone, D. 1995, ARA\&A, 33, 581

Kotilainen, J. K., Ward, M. J., Boisson, C., Depoy, D. L., \& Smith, M. G. 1992, MNRAS, 256, 149

Laine, S., Shlosman, I., Knapen, J. H., \& Peletier, R. F. 2002, ApJ, 567, 97

Larkin, J. E., Armus, L., Knop, R. A., Soifer, B. T., \& Matthews, K. 1998, ApJS, 114,59

Lepp, S., \& McCray, R. 1983, ApJ, 269, 560

Lester, D. F., Harvey, P. M., \& Carr, J. 1988, ApJ, 329, 641

Maciejewski, W. 2004, MNRAS, 354, 892

Magorrian, J., Tremaine, S., Richstone, D., et al. 1998, AJ, 115, 2285

Maiolino, R., Rieke, G. H., \& Rieke, M. J. 1996, AJ, 111, 537

Maiolino, R., Ruiz, M., Rieke, G. H., \& Papadopoulos, P. 1997, ApJ, 485, 552

Malkan, M. A., Gorjian, V., \& Tam, R. 1998, ApJS, 117, 25

Maloney, P. R., Hollenbach, D. J., \& Tielens, A. G. G. M. 1996, ApJ, 466, 561

Marconi, A., Moorwood, A. F. M., Salvati, M., \& Oliva, E. 1994, A\&A, 291, 18

Martini, P. 2004, in Coevolution of Black Holes and Galaxies, ed. L. C. Ho, 169

Martini, P., Regan, M. W., Mulchaey, J. S., \& Pogge, R. W. 2003, ApJ, 589, 774

Moran, E. C., Halpern, J. P., \& Helfand, D. J. 1996, ApJS, 106, 341

Mouri, H. 1994, ApJ, 427, 777

Mouri, H., \& Taniguchi, Y. 1992, ApJ, 386, 68

Nelson, C. H., MacKenty, J. W., Simkin, S. M., \& Griffiths, R. E. 1996, ApJ, 466, 713

Origlia, L., \& Oliva, E. 2000, A\&A, 357, 61

Origlia, L., Moorwood, A. F. M., \& Oliva, E. 1993, A\&A, 280, 536

Osterbrock, D. E. 1981, ApJ, 249, 462

Osterbrock, D. E. 1989, Astrophysics of gaseous nebulae and active galactic nuclei, Research supported by the University of California, John Simon Guggenheim Memorial Foundation, University of Minnesota, et al. Mill Valley, CA, University Science Books, 422

Page, M. J., Stevens, J. A., Mittaz, J. P. D., \& Carrera, F. J. 2001, Science, 294, 2516

Panessa, F., Wolter, A., Pellegrini, S., et al. 2005, ApJ, 631, 707

Pappa, A., Georgantopoulos, I., Ward, M., \& Zezas, A. L. 2002, MNRAS, 336, 714
Patsis, P. A. 2005, MNRAS, 358, 305

Polletta, M., Bassani, L., Malaguti, G., Palumbo, G. G. C., \& Caroli, E. 1996, ApJS, 106, 399

Prieto, M. A., Marco, O., \& Gallimore, J. 2005, MNRAS, 364, L28

Radford, S. J. E., Downes, D., \& Solomon, P. M. 1991, ApJ, 368, L15

Ramos Almeida, C., Pérez García, A. M., Acosta-Pulido, J. A., et al. 2006, ApJ, 645,148

Reunanen, J., Kotilainen, J. K., \& Prieto, M. A. 2003, MNRAS, 343, 192

Rigopoulou, D., Papadakis, I., Lawrence, A., \& Ward, M. 1997, A\&A, 327, 493

Rix, H.-W., Rieke, G., Rieke, M., \& Carleton, N. P. 1990, ApJ, 363, 480

Rodríguez-Ardila, A., Pastoriza, M. G., Viegas, S., Sigut, T. A. A., \& Pradhan, A. K. 2004, A\&A, 425, 457

Rodríguez-Ardila, A., Riffel, R., \& Pastoriza, M. G. 2005, MNRAS, 364, 1041

Rudy, R. J., \& Rodriguez-Espinosa, J. M. 1985, ApJ, 298, 614

Rudy, R. J., Cohen, R. D., \& Ake, T. B. 1988, ApJ, 332, 172

Sanders, D. B., Soifer, B. T., Elias, J. H., et al. 1988, ApJ, 325, 74

Schinnerer, E., Eckart, A., Tacconi, L. J., Genzel, R., \& Downes, D. 2000, ApJ, 533,850

Schlegel, D. J., Finkbeiner, D. P., \& Davis, M. 1998, ApJ, 500, 525

Schmitt, H. R., Kinney, A. L., Calzetti, D., \& Storchi Bergmann, T. 1997, AJ, 114,592

Shlosman, I., Frank, J., \& Begelman, M. C. 1989, Nature, 338, 45

Shlosman, I., Begelman, M. C., \& Frank, J. 1990, Nature, 345, 679

Solomon, P. M., \& Sage, L. J. 1988, ApJ, 334, 613

Solomon, P. M., Downes, D., \& Radford, S. J. E. 1992, ApJ, 398, L29

Solomon, P. M., Downes, D., Radford, S. J. E., \& Barrett, J. W. 1997, ApJ, 478, 144

Spergel, D. N., Verde, L., Peiris, H. V., et al. 2003, ApJS, 148, 175

Springel, V., Di Matteo, T., \& Hernquist, L. 2005, MNRAS, 361, 776

Sternberg, A., \& Dalgarno, A. 1989, ApJ, 338, 197

Tine, S., Lepp, S., Gredel, R., \& Dalgarno, A. 1997, ApJ, 481, 282

Tremaine, S., Gebhardt, K., Bender, R., et al. 2002, ApJ, 574, 740

Tutui, Y., Sofue, Y., Honma, M., Ichikawa, T., \& Wakamatsu, K.-i. 2000, PASJ, 52,803

Ulvestad, J. S., \& Wilson, A. S. 1984, ApJ, 278, 544

van Breugel, W. J. M., \& Dey, A. 1993, ApJ, 414, 563

Veilleux, S., \& Osterbrock, D. E. 1987, ApJS, 63, 295

Wallace, L., Livingston, W., Hinkle, K., \& Bernath, P. 1996, ApJS, 106, 165

Wallace, L., Meyer, M. R., Hinkle, K., \& Edwards, S. 2000, ApJ, 535, 325

Ward, M. J., Done, C., Fabian, A. C., Tennant, A. F., \& Shafer, R. A. 1988, ApJ, 324,767

Wolniewicz, L., Simbotin, I., \& Dalgarno, A. 1998, ApJS, 115, 293

Wyse, R. F. G. 2004, ApJ, 612, L17

Young, J. S., \& Scoville, N. Z. 1991, ARA\&A, 29, 581

Zuther, J., Eckart, A., Straubmeier, C., \& Voges, W. 2004, in AGN Physics with the Sloan Digital Sky Survey, ASP Conf. Ser., 311, 325

Zuther, J., Eckart, A., Voges, W., Bertram, T., \& Straubmeier, C. 2005, in Science with Adaptive Optics, Proceedings of the ESO Workshop Held at Garching, Germany, 16-19 September 2003, ed. W. Brandner, \& M. E. Kasper, Springer, 375

Zuther, J., Iserlohe, C., Pott, J.-U., Eckart, A., \& Voges, W. 2006, New Astron. Rev., 49, 508 